TRANSACTIONS OF THE

AMERICAN MATHEMATICAL SOCIETY

Volume 349, Number 10, October 1997, Pages 4107-4142

S 0002-9947(97)01849-7

\title{
ASYMPTOTIC ANALYSIS FOR LINEAR DIFFERENCE EQUATIONS
}

\author{
KATSUNORI IWASAKI
}

\begin{abstract}
We are concerned with asymptotic analysis for linear difference equations in a locally convex space. First we introduce the profile operator, which plays a central role in analyzing the asymptotic behaviors of the solutions. Then factorial asymptotic expansions for the solutions are given quite explicitly. Finally we obtain Gevrey estimates for the solutions. In a forthcoming paper we will develop the theory of cohomology groups for recurrence relations. The main results in this paper lay analytic foundations of such an algebraic theory, while they are of intrinsic interest in the theory of finite differences.
\end{abstract}

\section{INTRODUCTION}

Let $U$ be a locally convex linear space over $K=\mathbb{C}$ or $\mathbb{R}, P=\left(P_{n}\right)_{n \in \mathbb{Z}}$ an infinite sequence of linear operators $P_{n}: U \rightarrow U$. We are concerned with asymptotic analysis for the linear difference equation

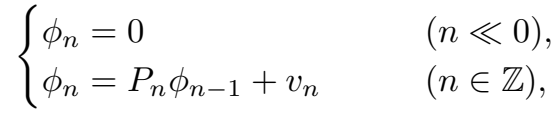

where the known data $v=\left(v_{n}\right)_{n \in \mathbb{Z}}$ is an infinite sequence of vectors in $U$ such that $v_{n}=0$ for all $n \ll 0$.

Let $V_{f}$ be the linear space of all infinite sequences $v=\left(v_{n}\right)_{n \in \mathbb{Z}}$ of vectors in $U$ such that $v_{n}=0$ for all $n \ll 0$. The space $V_{f}$ is referred to as the formal sequence space for $U$. Let $T: V_{f} \rightarrow V_{f}$ be the translation operator defined by

$$
(T v)_{n}=v_{n-1} \quad(n \in \mathbb{Z}) .
$$

We consider $P=\left(P_{n}\right)$ as a linear operator $P: V_{f} \rightarrow V_{f}$ defined by

$$
P v=\left(P_{n} v_{n}\right) \quad \text { for } \quad v=\left(v_{n}\right) \in V_{f} .
$$

A linear operator of this form is referred to as a homogeneous linear operator on $V_{f}$. Then the difference equation (1.1) is simply rewritten as

$$
(I-P T) \phi=v,
$$

where $I$ is the identity operator on $V_{f}$.

For any $v \in V_{f}$ it is clear that the difference equation (1.1) or (1.4) admits a unique solution $\phi \in V_{f}$ depending linearly on $v \in V_{f}$. This solution is denoted by

Received by the editors October 31, 1994 and, in revised form, March 18, 1996.

1991 Mathematics Subject Classification. Primary 39A10, 39A12, 40A05, 46M20.

Key words and phrases. Difference equation, profile operator, factorial asymptotic expansion, Gevrey estimate.

(C)1997 American Mathematical Society 
$\phi(v)=\left(\phi_{n}(v)\right)$ and called the formal solution for $v \in V_{f}$. Then $\phi: V_{f} \rightarrow V_{f}, v \mapsto$ $\phi(v)$ defines a linear operator, which is called the formal solution operator.

From the observation above, there is no difficulty in the difference equation (1.4) as long as it is considered on the formal sequence space $V_{f}$; there always exists a unique solution $\phi(v)=\left(\phi_{n}(v)\right) \in V_{f}$ for any $v \in V_{f}$, and there is no constraint on the asymptotic behavior of $\phi_{n}(v)$ as $n \rightarrow+\infty$. However, once (1.4) is considered on those linear subspaces $V$ of $V_{f}$ which are characterized by some global behaviors of $v=\left(v_{n}\right) \in V$ as $n \rightarrow+\infty$, the difference equation (1.4) becomes quite difficult; although there exists a unique formal solution $\phi(v) \in V_{f}$ for any $v \in V$, it is not at all clear whether $\phi(v)$ belongs to $V$ or not. Moreover the formal solution $\phi(v)$ for $v \in V$ might be subject to certain asymptotic conditions, but it is generally difficult to find such conditions effectively. This situation becomes clear if we consider, for example, the cases where $V$ is the linear space of all convergent sequences in $U$, or the space of all sequences $v=\left(v_{n}\right) \in V_{f}$ such that $\lim _{n \rightarrow+\infty} v_{n}=0$, etc.

The discussion above leads us to the problem of finding suitable pairs $(V, P)$ for which the difference equation (1.4) on the space $V$ can be analyzed in great detail. We introduce a nice class of such pairs $(V, P)$ in the following definition.

Definition 1.1. Let $V$ be a linear subspace of $V_{f}, P=\left(P_{n}\right)_{n \in \mathbb{Z}}$ a homogeneous linear operator on $V_{f}, N$ a nonnegative integer. Then the pair $(V, P)$ is said to be an asymptotic pair of order $N$ if the following conditions hold:

(AP-1) $P$ and $T$ map $V$ into itself,

(AP-2) for any $v=\left(v_{n}\right) \in V, v_{n} \rightarrow 0$ as $n \rightarrow+\infty$,

(AP-3) there exists a quartet $\left(c, U_{1}, \Phi,\left\{\Psi_{k}\right\}_{k=0}^{N}\right)$ such that

(1) $c \in K(=\mathbb{C}$ or $\mathbb{R})$,

(2) $U_{1}$ is a closed linear subspace of $U$,

(3) $\Phi: V \rightarrow U_{1}$ is a surjective linear operator,

(4) $\Psi_{k}: U_{1} \rightarrow U(k=0,1, \ldots, N)$ are linear operators, $\Psi_{0}$ is the inclusion map, and

(5) $\phi_{n}(v)$ admits the asymptotic expansion:

$\phi_{n}(v)=\left(\sum_{k=0}^{N} \Psi_{k}[n+c]_{k}\right) \Phi v+o\left(\frac{1}{n^{N}}\right) \quad$ as $n \rightarrow+\infty \quad(\forall v \in V)$,

where $[x]_{k}$ is the lower factorial monomial of degree $k$ (cf. Definition 2.2.1),

(AP-4) for any $v \in V, \phi(v) \in V_{f}$ belongs to $V$ if and only if $\Phi v=0$.

The operator $\Phi$ is called the profile operator. The triple $\mathcal{A}=\left(c, U_{1},\left\{\Psi_{k}\right\}_{k=0}^{N}\right)$ is referred to as the asymptotic data of $(V, P)$. Moreover the pair $(V, P)$ is said to be an asymptotic pair of order $\infty$ if there exists a quartet $\left(c, U_{1}, \Phi,\left\{\Psi_{k}\right\}_{k=0}^{\infty}\right)$ such that, for any nonnegative integer $N$, the pair $(V, P)$ is an asynptotic pair of order $N$ with profile operator $\Phi$ and asymptotic data $\left(c, U_{1},\left\{\Psi_{k}\right\}_{k=0}^{N}\right)$. The triple $\mathcal{A}=\left(c, U_{1},\left\{\Psi_{k}\right\}_{k=0}^{\infty}\right)$ is referred to as the asymptotic data of $(V, P)$.

We remark that the profile operator $\Phi$ is not a member of the asymptotic data $\mathcal{A}$. The reason for this will be mentioned just after Problem 1.2 below. In this paper we always assume $c=0$ for the sake of simplicity. This restriction causes no loss of generality since, if necessary, we may replace $n$ by $n+c$ without trouble. With this understanding the pair $\mathcal{A}=\left(U_{1},\left\{\Psi_{k}\right\}_{k=0}^{N}\right)$ or $\left(U_{1},\left\{\Psi_{k}\right\}_{k=0}^{\infty}\right)$ is referred to as 
the asymptotic data of $(V, P)$. If $(V, P)$ is an asymptotic pair, we may say that the conditions (AP), especially (AP-3) and (AP-4), provide good enough information about the difference equation (1.4) on the space $V$. The purpose of this paper is to settle the following problem.

Problem 1.2. (1) Construct asymptotic pairs $(V, P)$.

(2) Find algorithms for calculating the asymptotic data $\mathcal{A}$ explicitly only in terms of $P$.

In (2) of Problem 1.2 we do not require any algorithmic formula for the profile operator $\Phi$; expecting such a formula seems too easygoing. In fact, as mentioned previously, the linear subspace $V$ of $V_{f}$ should be characterized by some global behaviors of $v=\left(v_{n}\right) \in V$ as $n \rightarrow+\infty$, and therefore the space $V$ must be a highly transcendental object. Hence any asymptotic formula for the formal solution $\phi(v)$ for $v \in V$ cannot be purely algorithmic (nor algebraic); rather it must contain some transcendental terms.

So what we should try to do is to separate the asymptotic formula into two parts; one a transcendental part which perfectly reflects the global, and hence transcendental, nature of the space $V$; the other an algebraic part in which everything can be determined algorithmically in terms of $P$. Notice that, while $V$ is a transcendental object, $P$ is a formal, and hence algebraic, object since $P: V_{f} \rightarrow V_{f}$ makes sense on the formal space $V_{f}$. So the algebraic part should depend only on $P$. Now our basic idea is to consider that the profile operator $\Phi$ and the asymptotic data $\mathcal{A}$ represent the transcendental part and the algebraic part of the asymptotic formula (5) in (AP-3), respectively. This idea motivates (2) of Problem 1.2 as well as the separate treatment of the profile operator $\Phi$ from the asymptotic data $\mathcal{A}$. Finally, we remark that what is important about the profile operator $\Phi$ is its very existence, on the basis of which the entire theory works out.

In this paper we construct two classes of asymptotic pairs - rapidly decreasing pairs and Gevrey pairs. To do this we study the global behaviors of the solutions of the difference equation (1.4) as $n \rightarrow+\infty$ in fairly detail. The main results of this paper are rigorously stated in Section 3. In this introduction we confine ourselves to roughly sketching them by restricting our attention to asymptotic pairs of order $\infty$.

We assume that $P=\left(P_{n}\right)$ admits an upper factorial asymptotic expansion of the form

$$
P_{n} \sim \sum_{i=0}^{\infty} P^{(i)}(n)_{i} \quad \text { as } \quad n \rightarrow+\infty,
$$

where $P^{(i)}: U \rightarrow U$ are linear operators on $U$ and $(n)_{i}$ is the upper factorial monomial of degree $i$ (cf. Definition 2.2.1). See (3.4) for the rigorous meaning of (1.5). Let $\ell^{\infty}$ be the linear space of all infinite sequences $v=\left(v_{n}\right) \in V_{f}$ such that $v_{n} \rightarrow 0$ rapidly as $n \rightarrow+\infty$, i.e., the convergence is faster than any inverse polynomial order (cf. Definition 3.2.2). Then the formal solution $\phi(v)=\left(\phi_{n}(v)\right.$ ) for $v \in \ell^{\infty}$ of the difference equation (1.4) admits a lower factorial asymptotic expansion of the form

$$
\phi_{n}(v) \sim\left(\sum_{i=0}^{\infty} \Psi_{i}[n]_{i}\right) \Phi v \quad\left(\forall v \in \ell^{\infty}\right)
$$


(cf. Notation 3.4.1), where $\Phi: \ell^{\infty} \rightarrow U_{1}$ is a continuous linear operator of $\ell^{\infty}$ onto a closed subspace $U_{1}$ of $U$, and the coefficients $\Psi_{i}$ of the formal factorial series in (1.6) are continuous linear operators of $U_{1}$ into $U$. The subspace $U_{1}$ can be determined explicitly. Moreover an algorithm for determing $\Psi_{i}$ is obtained explicitly in terms of the coefficients $P^{(i)}$ of the asymptotic expansion (1.5) of $P$ (cf. Theorem II(2) and Definition 3.2.6). The asymptotic formula (1.6) implies that $\left(\ell^{\infty}, P\right)$ is an asymptotic pair of order $\infty$ with asymptotic data $\mathcal{A}=\left(U_{1},\left\{\Psi_{k}\right\}_{k=0}^{\infty}\right)$, the profile operator being $\Phi$ (cf. Corollary 3.4.2). The asymptotic pair $\left(\ell^{\infty}, P\right.$ ) is referred to as a rapidly decreasing pair.

Another and more important class of asymptotic pairs is obtained by the use of Gevrey spaces $\mathcal{G}^{t, a+}$ with appropriate Gevrey indices $(t, a)$. Here the Gevrey space $\mathcal{G}^{t, a+}$ is the linear space of all infinite sequences $v=\left(v_{n}\right) \in V_{f}$ such that, for any $b>a$ and for any continuous semi-norm $|\cdot|$ of $U$,

$$
\left|v_{n}\right|=O\left(\frac{b^{n}}{(n !)^{t}}\right) \quad \text { as } \quad n \rightarrow+\infty
$$

(cf. Definition 3.5.2). We show that if the index $(t, a)$ is chosen suitably then $\left(\mathcal{G}^{t, a+}, P\right)$ becomes an asymptotic pair. The admissible indices $(t, a)$ are determined explicitly in terms of the coefficients $P^{(i)}$ of the asymptotic expansion (1.5) of $P$ (cf. Corollary 3.6.2). The asymptotic pair $\left(\mathcal{G}^{t, a+}, P\right)$ is referred to as a Gevrey pair. Some examples of rapidly decreasing and Gevrey pairs are given in Section 7 .

Since we are interested in the asymptotic behavior of $\phi_{n}(v)$ as $n \rightarrow+\infty$, it causes no loss of generality to restrict our attention to the following truncated difference equation:

$$
\left\{\begin{array}{l}
\phi_{0}=v_{0}, \\
\phi_{n}=P_{n} \phi_{n-1}+v_{n} \quad\left(n \in \mathbb{Z}_{\geq 0}\right),
\end{array}\right.
$$

where the negative part $n \in \mathbb{Z}_{<0}$ of the difference equation (1.1) is truncated. In what follows we assume that the suffix $n$ ranges over the set $\mathbb{Z}_{\geq 0}$ of nonnegative integers. So $v=\left(v_{n}\right)$ and $\phi=\left(\phi_{n}\right)$ mean $v=\left(v_{n}\right)_{n \in \mathbb{Z}_{\geq 0}}$ and $\phi=\left(\phi_{n}\right)_{n \in \mathbb{Z}_{\geq 0}}$, respectively. Now the formal sequence space $V_{f}$ is redefined as

$$
V_{f}=\left\{v=\left(v_{n}\right)_{n \in \mathbb{Z}_{\geq 0}} ; v_{n} \in U\right\}
$$

The organization of this paper is as follows: In Section 2 we present some background materials from functional analysis and factorial series. This section is a preliminary to the later sections. In Section 3 we state the main results of this paper. In Section 4 we establish the existence of the profile operator $\Phi$. In Section 5 we derive a factorial asymptotic expansion for the solutions $\phi(v)$ of the difference equation (1.8). Section 6 is devoted to the Gevrey estimates which enable us to construct the Gevrey pairs. In the final Section 7, we apply our results to some difference equations which arise from systems of confluent hypergeometric differential equations in two variables. This demonstrates the applicability of our results to a rather different and unexpected area of mathematics.

\section{ACKNOWLEDGEMENT}

The author would like to thank Professor Hideyuki Majima and Ms. Sumiko Ishizuka for various discussions. This work was done while the author was a visitor at I.R.M.A., Université Louis Pasteur de Strasbourg. Thanks are also due to Professor Raymond Gérard for his kind hospitality. 


\section{Preliminaries}

2.1. Functional analysis. Let $U$ be a locally convex linear space, $\mathcal{N}$ any fixed system of semi-norms on $U$ which determines the locally convex topology of $U$. For our purpose it is desirable that $\mathcal{N}$ is as small as possible as a set.

Definition 2.1.1. A linear transformation $A$ of $U$ is said to be strongly bounded if, for any $|\cdot| \in \mathcal{N}$, there exists a constant $C$ depending on $|\cdot|$ such that

$$
|A u| \leq C|u| \quad(\forall u \in U) .
$$

We denote by $|A|$ the infimum of all such constants $C$. Let $\mathcal{B}=\mathcal{B}(U)$ be the set of all strongly bounded transformations of $U$. Then $\mathcal{B}$ is a locally convex algebra with system of semi-norms $\mathcal{N}$ such that

$$
|A B| \leq|A||B| \quad(\forall A, B \in \mathcal{B}, \forall|\cdot| \in \mathcal{N}) .
$$

Remark 2.1.2. Let $X$ and $Y$ be supplementary projections in $\mathcal{B}$, i.e., $X \in \mathcal{B}, X^{2}=$ $X$ and $X+Y=I$. For each $|\cdot| \in \mathcal{N}$ we can define a semi-norm $\|\cdot\|$ on $U$ by

$$
\|u\|=\sqrt{|X u|^{2}+|Y u|^{2}} .
$$

Note that $|\cdot|$ and $\|\cdot\|$ are equivalent semi-norms. Indeed, we have

$$
\frac{1}{\sqrt{2}}|u| \leq\|u\| \leq \sqrt{|X|^{2}+|Y|^{2}}|u|
$$

Hence, without loss of generality, we may assume

$$
|u|^{2}=|X u|^{2}+|Y u|^{2} \quad(\forall|\cdot| \in \mathcal{N}) .
$$

For, otherwise, we can replace $|\cdot|$ by $\|\cdot\|$; the latter satisfies (2.1). If $X$ and $Y$ are given then we always assume (2.1). Under this assumption we have

$$
|X|,|Y|=0 \text { or } 1 .
$$

The use of the Landau symbol $O$ on a locally convex space is sometimes confusing. So we should clarify in what sense the symbol $O$ is used in this paper.

Notation 2.1.3. (i) On a normed space: Let $S$ be a normed space with the norm $|\cdot|$, e.g., $S=\mathbb{C}$ or $\mathbb{R}$, etc. Let $\left\{s_{i}\right\}_{i \in I}$ be a subset of $S,\left\{b_{i}\right\}_{i \in I}$ a set of nonnegative numbers, both indexed by $i \in I$. Then we write

$$
a_{i}=O\left(b_{i}\right) \quad(i \in I)
$$

if there exists a constant $C$ such that $\left|a_{i}\right| \leq C b_{i}$ for all $i \in I$. There is no ambiguity in this case, since the norm $|\cdot|$ is unique.

(ii) On a locally convex space: Let $S$ be a locally convex space with system of semi-norms $\mathcal{N}$, e.g., $S=U, \mathcal{B}$ or any other locally convex spaces that appear later. Let $\left\{s_{i}\right\}_{i \in I}$ be a subset of $S,\left\{b_{i}\right\}_{i \in I}$ a set of nonnegative numbers. Then it is possible to provide the following two meanings for the expression

$$
s_{i}=O\left(b_{i}\right) \quad(i \in I) .
$$

(1) For each semi-norm $|\cdot| \in \mathcal{N}$ there exists a constant $C$ such that $\left|s_{i}\right| \leq C b_{i}$ for all $i \in I$, where $C$ may depend on $|\cdot|$.

(2) There exists a constant $C$ such that $\left|s_{i}\right| \leq C b_{i}$ for all $i \in I$ and $|\cdot| \in \mathcal{N}$, where $C$ is independent of $|\cdot|$. 
Clearly (2) implies (1), but the converse is not always true if $\mathcal{N}$ is an infinite set. In this paper the expression (2.3) means (1). For (2) we use the following expression:

$$
s_{i}=O_{u}\left(b_{i}\right) \quad(i \in I) .
$$

The subscript $u$ stands for the uniformity with respect to the semi-norms.

(iii) Let $S$ be as in (ii), $a_{i}(|\cdot|)$ and $b_{i}(|\cdot|)$ nonnegative numbers depending on $i \in I$ and $|\cdot| \in \mathcal{N}$. First we write

$$
a_{i}(|\cdot|)=O\left(b_{i}(|\cdot|)\right) \quad(i \in I),
$$

if, for each $|\cdot| \in \mathcal{N}$, there exists a constant $C$ depending on $|\cdot|$ such that $a_{i}(|\cdot|) \leq$ $C b_{i}(|\cdot|)$ for all $i \in I$. Secondly we write

$$
a_{i}(|\cdot|)=O_{u}\left(b_{i}(|\cdot|)\right) \quad(i \in I),
$$

if there exists a constant $C$ independent of $|\cdot|$ such that $\left.a_{i}(|\cdot|) \leq C b_{i}(|\cdot|)\right)$ for all $i \in I$ and $|\cdot| \in \mathcal{N}$.

The above notation is quite convenient in what follows.

\subsection{Factorial series.}

Definition 2.2.1. The upper factorial monomial of degree $i$ is defined by

$$
(n)_{i}=\frac{(-1)^{i}(i-1) !}{n(n+1)(n+2) \cdots(n+i-1)} \quad(i \geq 1) .
$$

Similarly the lower factorial monomial of degree $i$ is defined by

$$
[n]_{i}=\frac{(-1)^{i}(i-1) !}{n(n-1)(n-2) \cdots(n-i+1)} \quad(i \geq 1) .
$$

By convention we set $(n)_{0}=[n]_{0}=1$. For a linear space $S$, an upper (resp. a lower) factorial series over $S$ is an expression of the form

$$
\sum_{i=0}^{\infty} s_{i}(n)_{i} \quad\left(\operatorname{resp} . \quad \sum_{i=0}^{\infty} s_{i}[n]_{i}\right),
$$

where $s_{i} \in S$. An upper (resp. a lower) factorial polynomial over $S$ is an upper (resp. a lower) factorial series such that $s_{i} \neq 0$ for at most finitely many $i$.

In this paper a factorial series is always a formal factorial series; the adjective "formal" is omitted. If $S$ is a ring then the set of all upper (or lower) factorial series admits a ring structure. We refer to [3][6][12][13] for more detailed information about factorial series.

Notation 2.2.2.

$$
\begin{aligned}
{\left[\begin{array}{l}
i \\
j
\end{array}\right] } & =\frac{i(i+1)(i+2) \cdots(i+j-1)}{j !} & (j \geq 1), \\
{[i, j] } & =\frac{(i-1) !(j-1) !}{(i+j-1) !} & (i, j \geq 1) .
\end{aligned}
$$

By convention we set

$$
\left[\begin{array}{l}
i \\
0
\end{array}\right]=[j, 0]=[0, k]=1 \quad(j, k \geq 0) .
$$

The following lemmas are used in the later sections. 
Lemma 2.2.3. For any $i \geq 1$,

$$
[n]_{i}-[n-1]_{i}=[n]_{i+1} .
$$

Lemma 2.2.4. For any $N \geq i \geq 1$,

$$
[n+m]_{i}=\sum_{j=i}^{N}\left[\begin{array}{c}
m \\
j-i
\end{array}\right][n]_{j}+O\left(\frac{1}{n^{N+1}}\right) \quad(n \geq N) .
$$

Lemma 2.2.5. For any $N \geq i+j \geq 1$,

$$
\begin{aligned}
(n)_{i}[n-1]_{j} & =[i, j][n+i-1]_{i+j} \\
& =[i, j] \sum_{k=i+j}^{N}\left[\begin{array}{c}
i-1 \\
k-i-j
\end{array}\right][n]_{k}+O\left(\frac{1}{n^{N+1}}\right) \quad(n \geq N) .
\end{aligned}
$$

The proof of these lemmas is omitted, since it is rather standard.

\section{Main Results}

3.1. Assumptions. In the statement of the main results we make the following assumptions.

Assumption A. $U$ is a sequentially complete locally convex space. $P=\left(P_{n}\right)$ is an infinite sequence in $\mathcal{B} . X$ and $Y$ are supplementary projections in $\mathcal{B}$.

$$
\begin{aligned}
& X P_{n} X=X+O\left(\frac{1}{n^{2}}\right), \quad X P_{n} Y=O\left(\frac{1}{n}\right), \\
& Y P_{n} X=O\left(\frac{1}{n}\right) \quad(n \geq 1),
\end{aligned}
$$

and, for each $|\cdot| \in \mathcal{N}$, there exists a constant $\rho(0 \leq \rho<1)$ such that

$$
\left|Y P_{n} Y\right| \leq \rho+O\left(\frac{1}{n^{2}}\right) \quad(n \geq 1) .
$$

The constant $\rho$ may depend on the semi-norm $|\cdot| \in \mathcal{N}$.

Assumption $\mathbf{A}_{u}$. All assumptions are the same as in Assumption A except for the following alteration: The Landau symbol $O$ in (3.1) and (3.2) is replaced by $O_{u}$ (cf. Notation 2.1.3), and the constant $\rho$ in (3.2) is independent of $|\cdot| \in \mathcal{N}$.

Remark 3.1.1. If $U$ is sequentially complete then $\mathcal{B}$ is also sequentially complete.

Assumption $\mathbf{B}_{N}$. $N$ is a nonnegative integer. $U$ is a sequentially complete locally convex space. $P=\left(P_{n}\right)$ is an infinite sequence in $\mathcal{B}$. $X$ and $Y$ are supplementary projections in $\mathcal{B}$. There exist strongly bounded operators $P^{(i)} \in \mathcal{B}(i=0,1, \ldots, N+$ 1) such that

$$
P_{n}=\sum_{i=0}^{N+1} P^{(i)}(n)_{i}+O\left(\frac{1}{n^{N+2}}\right) \quad(n \geq N+1)
$$

in the space $\mathcal{B}$, and

(1) $X P^{(0)}=P^{(0)} X=X$,

(2) for each $|\cdot| \in \mathcal{N},|Z|<1$, where $Z:=Y P^{(0)} Y$, and

(3) $X P^{(1)} X=O$.

In case $N=\infty$ the assumption must be sligntly modified. 
Assumption $\mathbf{B}_{\infty}$. All assumptions are the same as in Assumption $\mathrm{B}_{N}$ except for (3.3). Condition (3.3) is replaced by the following one: There exist $P^{(i)} \in \mathcal{B}(i \geq 0)$ such that (3.3) holds for all $N \geq 0$. We express this condition as

$$
P_{n} \sim \sum_{i=0}^{\infty} P^{(i)}(n)_{i} .
$$

3.2. Notation. In order to state the main results we introduce some notation.

Notation 3.2.1. We set

$$
U_{\nu}=\{u \in U ; X u=\nu u\} \quad(\nu=0,1) .
$$

Then $U_{\nu}(\nu=0,1)$ are closed linear subspaces of $U$ such that $U=U_{1} \oplus U_{0}$. We denote by $I_{\nu}$ the identity map on $U_{\nu}$.

Definition 3.2.2. For each $|\cdot| \in \mathcal{N}$ and $v=\left(v_{n}\right) \in V_{f}$, we set

$$
\begin{aligned}
|v|_{0} & =\sum_{n=0}^{\infty}(n+1)\left|X v_{n}\right|+\sup _{n \geq 0}\left\{(n+1)\left|Y v_{n}\right|\right\}, \\
|v|_{N} & =\sup _{n \geq 0}(n+1)^{N+2}\left|X v_{n}\right|+\sup _{n \geq 0}\left\{(n+1)^{N+1}\left|Y v_{n}\right|\right\} \quad(N \geq 1) .
\end{aligned}
$$

Let $\ell^{N}$ be the linear space of all $v \in V_{f}$ such that $|v|_{N}<\infty$ for all $|\cdot| \in \mathcal{N}$. Note that $\ell^{N}$ is a locally convex space having semi-norms $|\cdot|_{N}$ with $|\cdot| \in \mathcal{N}$, and that there exists a bounded inclusion $\ell^{N+1} \hookrightarrow \ell^{N}$. Moreover we set

$$
\ell^{\infty}=\bigcap_{N \geq 0} \ell^{N}
$$

Then $\ell^{\infty}$ is a locally convex space having semi-norms $|\cdot|_{N}$ with $|\cdot| \in \mathcal{N}$ and $N \in \mathbb{Z}_{\geq 0}$.

Definition 3.2.3. For $m \in \mathbb{Z}_{\geq 0}$ let $\iota_{m}$ be the bounded linear map defined by

$$
\iota_{m}: U_{1} \rightarrow \ell^{0}, \quad u \mapsto\left(\delta_{n m} u\right)_{n \geq 0},
$$

where $\delta_{n m}$ is the Kronecker symbol.

Notation 3.2.4. Using Notation 2.2.2, we set

$$
P_{i j}=\sum_{k=1}^{i-j}[j, k]\left[\begin{array}{c}
k-1 \\
i-j-k
\end{array}\right] P^{(k)} \quad(0 \leq j<i \leq N+1) .
$$

For example, for $1 \leq i-j \leq 3$, we have

$$
\begin{aligned}
P_{i+1, i} & =\frac{P^{(1)}}{i_{+}}, \quad P_{i+2, i}=\frac{P^{(2)}}{i_{+}(i+1)}, \\
P_{i+3, i} & =\frac{P^{(2)}}{i_{+}(i+1)}+\frac{P^{(3)}}{i_{+}(i+1)(i+2)} \quad(i \geq 0),
\end{aligned}
$$

where $i_{+}=\max \{i, 1\}$.

Notation 3.2.5. Since $|Z|<1$ for any $|\cdot| \in \mathcal{N}$ (cf. (2) of Assumption $\mathrm{B}_{N}$ ), the sequential completeness of $\mathcal{B}$ (cf. Remark 3.1.1) implies that $I_{0}-Z: U_{0} \rightarrow U_{0}$ has an inverse in $\mathcal{B}\left(U_{0}\right)$, which is denoted by $\left(I_{0}-Z\right)^{-1}$. Indeed the inverse map is given by the C. Neumann series $\sum_{n=0}^{\infty} Z_{0}^{n}$. We set

$$
A_{i j}=X P_{i+1, j}+\left(I+\frac{1}{i} X P^{(1)}\right)\left(I_{0}-Z\right)^{-1}\left(Y P_{i j}-\delta_{i, j+1} Z\right) \quad(0 \leq j<i \leq N),
$$

where $\delta_{i j}$ is the Kronecker symbol. 
Definition 3.2.6. For any $i \in \mathbb{Z}_{\geq 2}$ let $\mathbf{S}_{i}$ be the set of all subsets of $\{1,2, \ldots, i-1\}$ (including the empty set $\emptyset$ ). For each $J \in \mathbf{S}_{i}$ we set

$$
A_{J}= \begin{cases}A_{i 0} & (J=\emptyset), \\ A_{i j_{k}} A_{j_{k} j_{k-1}} \cdots A_{j_{2} j_{1}} A_{j_{1} 0} & (J \neq \emptyset),\end{cases}
$$

where $J=\left\{j_{1}, j_{2}, \ldots, j_{k}\right\}$ with $j_{1}<j_{2}<\cdots<j_{k}$ in the latter case. We define the operators $\Psi_{i} \in \mathcal{B}(i=0,1, \ldots, N)$ by $\Psi_{0}=I, \Psi_{1}=A_{10}$ and

$$
\Psi_{i}=\sum_{J \in \mathbf{S}_{i}} A_{J} \quad(i \geq 2) .
$$

Lemma 3.2.7. The operators $\Psi_{i}(i=0,1, \ldots, N)$ are determined by $\Psi_{0}=I$ and the recurrence relation

$$
\Psi_{i}=\sum_{j=0}^{i-1} A_{i j} \Psi_{j} \quad(1 \leq i \leq N) .
$$

This lemma is easily established by induction on $i$.

Remark 3.2.8. For $i=1,2, \ldots, N$, the operator $\Psi_{i}$ depends only on the first $(j+2)$ coefficients $P^{(0)}, P^{(1)}, \ldots, P^{(i+1)}$ of $P$ (cf. (3.3)).

\subsection{Profile operator.}

Theorem I. (1) Under Assumption A, there exist a bounded linear operator $\Phi$ : $\ell^{0} \rightarrow U_{1}$ such that

$$
\left|\phi_{n}(v)-\Phi v\right|=O\left(\frac{|v|_{0}}{n+1}\right) \quad\left(v \in \ell^{0}, n \geq 0\right) .
$$

(2) Under Assumption $\mathrm{A}_{u}$, the Landau symbol $O$ in (3.5) can be replaced by $O_{u}$ (cf. Notation 2.1.3). Moreover there exists an integer $m_{0}$ such that $\Phi \circ \iota_{m}: U_{1} \rightarrow U_{1}$ is a topological isomorphism for all $m \geq m_{0}$ (cf. Definition 3.2.3).

Remark 3.3.1. The operator $\Phi$ is called the profile operator (cf. Definition 4.8.1). The author does not know whether Assertion (2) of Theorem I holds under Assumption A (cf. Remark 4.8.4). This assertion is closely related to Condition (3) of (AP-3) in Definition 1.1. So we use it essentially to construct asymptotic pairs.

Theorem I is established in 4.8 (cf. Theorem 4.8.3).

\subsection{Asymptotic expansions.}

Theorem II. Let $\Phi$ be the profile operator in Theorem I, $\Psi_{i}$ the operators defined by Definition 3.2.6.

(1) Under Assumption $\mathrm{B}_{N}$ we have

$$
\left|\phi_{n}(v)-\left(\sum_{i=0}^{N} \Psi_{i}[n]_{i}\right) \Phi v\right|=O\left(\frac{|v|_{N}}{(n+1)^{N+1}}\right) \quad\left(v \in \ell^{N}, n \geq N\right) .
$$

(2) Under Assumption $\mathrm{B}_{\infty}$, for any $N \geq 0$, we have

$$
\left|\phi_{n}(v)-\left(\sum_{i=0}^{N} \Psi_{i}[n]_{i}\right) \Phi v\right|=O\left(\frac{|v|_{N}}{(n+1)^{N+1}}\right) \quad\left(v \in \ell^{\infty}, n \geq N\right) .
$$


Notation 3.4.1. Regarding $v \mapsto \phi_{n}(v)$ as an operator $\phi_{n}: \ell^{\infty} \rightarrow U$, we express Assertion (2) of Theorem II as

$$
\phi_{n} \sim\left(\sum_{i=0}^{\infty} \Psi_{i}[n]_{i}\right) \Phi \quad \text { in } \ell^{\infty} .
$$

Theorem II is established in Section 5 (cf. Theorem 5.1.1). From Theorem I and Theorem II we obtain the following corollary.

Corollary 3.4.2. Under Assumptions $\mathrm{A}_{u}$ and $\mathrm{B}_{\infty},\left(\ell^{\infty}, P\right)$ is an asymptotic pair of order $\infty$. The asymptotic data of $\left(\ell^{\infty}, P\right)$ is given by $\left(U_{1},\left\{\Psi_{i}\right\}_{i=0}^{\infty}\right)$, where $U_{1}$ and $\Psi_{i}$ are defined in Notation 3.2.1 and Definition 3.2.6, respectively.

Corollary 3.4.2 is established in 5.5.

3.5. Gevrey spaces. We introduce Gevrey spaces and establish some notation.

Definition 3.5.1. Let $t \geq 0$ and $a>0$. For $v=\left(v_{n}\right) \in V_{f}$ and $|\cdot| \in \mathcal{N}$ we set

$$
|v|_{t, a}=\sup _{n \geq 0}\left\{\frac{(n !)^{t}}{a^{n}}\left|v_{n}\right|\right\} \text {. }
$$

Let $\mathcal{G}^{t, a}$ be the set of all $v \in V_{f}$ such that $|v|_{t, a}<\infty$ for all $|\cdot| \in \mathcal{N}$. Then $\mathcal{G}^{t, a}$ is a locally convex space having semi-norms $|\cdot|_{t, a}$ with $|\cdot| \in \mathcal{N}$. $\mathcal{G}^{t, a}$ is called the Gevrey space with index $(t, a)$. An index $(t, a)$ is said to be of convergent type if either $t=0,0<a<1$ or $t>0, a>0$ holds.

Definition 3.5.2. Notice that if $a<b$ then $\mathcal{G}^{t, a} \subset \mathcal{G}^{t, b}$. For $t, a \geq 0$ we set

$$
\mathcal{G}^{t, a+}=\bigcap_{b>a} \mathcal{G}^{t, b}
$$

Then $\mathcal{G}^{t, a+}$ is a locally convex space having semi-norms $|\cdot|_{t, b}$ with $|\cdot| \in \mathcal{N}$ and $b>a . \mathcal{G}^{t, a+}$ is called the Gevrey space with index $(t, a+)$. An index $(t, a+)$ is said to be of convergent type if either $t=0,0 \leq a<1$ or $t>0, a \geq 0$ holds.

Any Gevrey space $\mathcal{G}^{t, a}$ (resp. $\mathcal{G}^{t, a+}$ ) of convergent type is contained in $\ell^{\infty}$, and hence the profile operator $\Phi$ acts on $\mathcal{G}^{t, a}$ (resp. $\mathcal{G}^{t, a+}$ ). Thus we can introduce the following spaces.

Definition 3.5.3. For any index $(t, a)$ or $(t, a+)$ of convergent type, we set

$$
\mathcal{G}_{0}^{t, a}=\left\{v \in \mathcal{G}^{t, a} ; \Phi v=0\right\}, \quad \mathcal{G}_{0}^{t, a+}=\left\{v \in \mathcal{G}^{t, a+} ; \Phi v=0\right\} .
$$

In addition to Assumption $\mathrm{B}_{N}$ we make the following assumption.

Assumption C. Let $p, q$ and $r$ be integers such that $1 \leq p, q \leq N+2$ and $0 \leq$ $r \leq N+2$. We assume

$$
\begin{aligned}
& X P^{(i)} Y=O \quad(0 \leq i<p), \quad Y P^{(i)} X=O \quad(0 \leq i<q), \\
& Y P^{(i)} Y=O \quad(0 \leq i<r) .
\end{aligned}
$$

If $r=0$ then the third condition should be ignored.

Notation 3.5.4. Let $p, q, r$ be as in Assumption C. The following integer $s$ plays an important role in what follows:

$$
s=\min \{p+q-1, r\} .
$$


Definition 3.5.5. Under Assumptions $\mathrm{B}_{N}$ and $\mathrm{C}$, for each $|\cdot| \in \mathcal{N}$, we introduce the following constants:

$$
\begin{array}{ll}
c_{1}(|\cdot|)=\varlimsup_{n \rightarrow \infty}\left\{n(n+1)\left|X P_{n} X-X\right|\right\}, & c_{2}(|\cdot|)=\varlimsup_{n \rightarrow \infty}\left\{n^{p}\left|X P_{n} Y\right|\right\}, \\
c_{3}(|\cdot|)=\varlimsup_{n \rightarrow \infty}\left\{n^{q}\left|Y P_{n} X\right|\right\}, & c_{4}(|\cdot|)=\varlimsup_{n \rightarrow \infty}\left\{n^{r}\left|Y P_{n} Y\right|\right\} .
\end{array}
$$

By Assumptions $\mathrm{B}_{N}$ and $\mathrm{C}$, they are well-defined finite numbers. Using these constants, we define the constant $c_{0}(|\cdot|)$ by

$$
c_{0}(|\cdot|)= \begin{cases}c_{2}(|\cdot|) c_{3}(|\cdot|) & (p+q-1<r), \\ c_{2}(|\cdot|) c_{3}(|\cdot|)+c_{4}(|\cdot|) & (p+q-1=r), \\ c_{4}(|\cdot|) & (p+q-1>r) .\end{cases}
$$

Finally we introduce the following (possibly infinite) constant:

$$
a_{0}=\sup \left\{c_{0}(|\cdot|) ;|\cdot| \in \mathcal{N}\right\} .
$$

Remark 3.5.6. In the following cases, (3.3) in Assumption $\mathrm{B}_{N}$ implies

$$
\begin{aligned}
& c_{1}(|\cdot|)=\left|X P^{(2)} X\right| \quad(N \geq 1), \\
& c_{2}(|\cdot|)=(p-1) !\left|X P^{(p)} Y\right| \quad(1 \leq p \leq N+1) \text {, } \\
& c_{3}(|\cdot|)=(q-1) !\left|Y P^{(q)} X\right| \quad(1 \leq q \leq N+1) \text {, } \\
& c_{4}(|\cdot|)= \begin{cases}|Z| & (r=0), \\
(r-1) !\left|Y P^{(r)} Y\right| & (1 \leq r \leq N+1) .\end{cases}
\end{aligned}
$$

If $r=0$ then $p+q-1 \geq 1>0=r$, and hence $c_{0}(|\cdot|)=c_{4}(|\cdot|)=|Z|$. In this case, (2) of Assumption $\mathrm{B}_{N}$ implies $c_{0}(|\cdot|)<1$.

\subsection{Gevrey estimates.}

Theorem III. Under Assumptions $\mathrm{B}_{N}$ and $\mathrm{C}$, if the integer s (cf. Notation 3.5.4) and the index $(t, a)$ satisfy one of the following conditions:

(1) $t=s=0, c_{0}(|\cdot|)<a<1$ (cf. Remark 3.5.6),

(2) $t=0<s, 0<a<1$,

(3) $0<t<s, a>0$,

(4) $t=s>0, a>c_{0}(|\cdot|)$,

then there exists a nonnegative constant $M(t, a,|\cdot|)$, depending only on $(t, a)$ and $|\cdot| \in \mathcal{N}$, such that

$$
\left|\phi_{n}(v)\right| \leq M(t, a,|\cdot|) \frac{(n+1)^{p+1} a^{n}}{(n !)^{t}}|v|_{t, a} \quad\left(v \in \mathcal{G}_{0}^{t, a}, n \geq 0\right),
$$

where $\mathcal{G}_{0}^{t, a}$ is defined in Definition 3.5.3.

Theorem III is established in 6.6 (cf. Theorem 6.6.2).

Corollary 3.6.1. Under Assumptions $\mathrm{B}_{N}$ and $\mathrm{C}$, if $\left(s, a_{0}\right)$ (cf. Notation 3.5.4 and Definition 3.5.5) and $(t, a)$ satisfy one of the following conditions:

(1) $t=s=0, a_{0} \leq a<1$,

(2) $t=0<s, 0 \leq a<1$,

(3) $0<t<s, a \geq 0$,

(4) $t=s>0, a_{0}<\infty$ and $a \geq a_{0}$, 
then the linear map

$$
\phi: \mathcal{G}_{0}^{t, a+} \rightarrow \mathcal{G}^{t, a+}, \quad v \mapsto \phi(v)
$$

is well-defined and bounded, where $\mathcal{G}_{0}^{t, a+}$ is defined in Definition 3.5.3.

Corollary 3.6.1 immediately follows from Theorem III. Moreover we have the following corollary.

Corollary 3.6.2. Under Assumptions $\mathrm{A}_{u}, \mathrm{~B}_{N}$ and $\mathrm{C}$, if one of the conditions (1)(4) in Corollary 3.6.1 holds, then the pair $\left(\mathcal{G}^{t, a+}, P\right)$ is an asymptotic pair of order $N$. The asymptotic data of $\left(\mathcal{G}^{t, a+}, P\right)$ is given by $\left(U_{1},\left\{\Psi_{i}\right\}_{i=0}^{N}\right)$, where $U_{1}$ and $\Psi_{i}$ are defined in Notation 3.2.1 and Definition 3.2.6, respectively.

Corollary 3.6.2 is established in 6.7. We refer to Remark 3.3.1 for the reason why Assumption $\mathrm{A}_{u}$ is added to Assumptions $\mathrm{B}_{N}$ and $\mathrm{C}$ in Corollary 3.6.2.

\section{Profile Operator}

\subsection{Propagators.}

Definition 4.1.1. We set $P_{m m}=I(m \geq 0)$ and

$$
P_{n m}=P_{n} P_{n-1} \cdots P_{m+2} P_{m+1} \quad(n>m \geq 0) .
$$

$P_{n m}$ is called the propagator (from time $m$ to time $n$ ).

Lemma 4.1.2. For any $v=\left(v_{n}\right) \in V_{f}$ the solution $\phi(v)$ of the difference equation (1.8) is given by

$$
\phi_{n}(v)=\sum_{m=0}^{n} P_{n m} v_{m}
$$

Theorem 4.1.3. Under Assumption A we have

$$
\begin{aligned}
X\left(P_{k m}-P_{n m}\right) X=O\left(\frac{1}{n+1}\right) & (k \geq n \geq m \geq 0), \\
X\left(P_{k m}-P_{n m}\right) Y=O\left(\frac{1}{n+1}\left(\rho^{n-m}+\frac{1}{m+1}\right)\right) & (k \geq n \geq m \geq 0), \\
Y P_{n m} X=O\left(\frac{1}{n+1}\right) & (n \geq m \geq 0), \\
Y P_{n m} Y=O\left(\rho^{n-m}+\frac{1}{(n+1)(m+1)}\right) & (n \geq m \geq 0) .
\end{aligned}
$$

Under Assumption $\mathrm{A}_{u}$ the Landau symbol $O$ can be replaced by $O_{u}$.

Remark 4.1.4. For a sufficiently large fixed $m$, many authors have studied asymptotic representations of $P_{n m}$ with respect to $n$ (cf. [1][2][4][5][11]). In their studies $U$ is assumed to be a finite-dimensional complex or real vector space. In this paper, however, we need an asymptotic representation with respect to both $n$ and $m$. Theorem 4.1.3 is by no means the best possible result; it is just simple and sufficient for our purpose. After some preliminaries, Theorem 4.1.3 will be established in 4.6. 
4.2. Two-dimensional problem. Theorem 4.1 .3 will be established by the method of majorants. In order to construct the majorants, we consider the following two-dimensional problem: Let

$$
M_{n}=\left(\begin{array}{cc}
1 & \frac{a}{n+1} \\
\frac{b}{n+1} & \rho
\end{array}\right)+O\left(\frac{1}{(n+1)^{2}}\right) \quad(n \geq 0),
$$

where $a, b>0$ and $0 \leq \rho<1$. Put $M_{m m}=I(m \geq 0)$ and

$$
M_{n m}=M_{n} M_{n-1} \cdots M_{m+1} \quad(n>m \geq 0) .
$$

The problem is to obtain an asymptotic representation of $M_{n m}$ with respect to $n$ and $m$.

We follow the method of Z. Benzaid and D.A. Lutz [1] with some additional elaboration. We set

$$
S_{n}=\left(\begin{array}{cc}
1 & -\frac{\alpha}{n+1} \\
\frac{\beta}{n+1} & 1
\end{array}\right) \quad(n \geq 0)
$$

where $\alpha=\frac{a}{1-\rho}$ and $\beta=\frac{b}{1-\rho}$. Moreover we set $N_{n}=\left(S_{n}\right)^{-1} M_{n} S_{n-1}$. Then we have $N_{n}=D+E_{n}$, where

$$
D=\left(\begin{array}{ll}
1 & 0 \\
0 & \rho
\end{array}\right), \quad E_{n}=O\left(\frac{1}{(n+1)^{2}}\right) \quad(n \geq 1) .
$$

We set $N_{m m}=I(m \geq 0)$ and $N_{n m}=N_{n} N_{n-1} \cdots N_{m+1}(n>m \geq 0)$. Then $M_{n m}=S_{n} N_{n m}\left(S_{m}\right)^{-1}$. So the problem is reduced to the same problem for $N_{n m}$.

4.3. Contraction mapping. For each fixed $m \geq 0$, let $\mathcal{L}$ be the linear space of all sequences $L=\left(L_{n}\right)_{n \geq m}$ in $M_{2}(\mathbb{R})$. We define a linear transformation $K: \mathcal{L} \rightarrow \mathcal{L}$ by $(K L)_{m}=O$ and

$$
(K L)_{n}=\sum_{i=m+1}^{n} D^{n-i} E_{i} L_{i-1} \quad(n>m)
$$

where $D$ and $E_{i}$ are given by (4.2). Moreover we define $N^{(m)}, D^{(m)} \in \mathcal{L}$ by

$$
N^{(m)}=\left(N_{n m}\right)_{n \geq m}, \quad D^{(m)}=\left(D^{n-m}\right)_{n \geq m} .
$$

Lemma 4.3.1. (1) For any $\Lambda \in \mathcal{L}$ the equation (4.5) below has a unique solution $L(\Lambda) \in \mathcal{L}:$

$$
L=\Lambda+K L
$$

(2) $N^{(m)}=L\left(D^{(m)}\right)$, where $N^{(m)}$ and $D^{(m)}$ are given by (4.4).

Proof. Assertion (1) follows from the fact that $K$ is an operator of Volterra type. Assertion (2) is a consequence of the very definition of $K$ (not immediate).

Let $\mathcal{L}^{\infty}$ be the linear subspace of $\mathcal{L}$ consisting of all $L=\left(L_{n}\right) \in \mathcal{L}$ such that $\|L\|:=\sup _{n \geq m}\left|L_{n}\right|<\infty$, where $|\cdot|$ is the operator norm in $M_{2}(\mathbb{R})$. We set

$$
c_{m}=\sum_{i=m+1}^{\infty}\left|E_{i}\right| \text {. }
$$

Since $E_{i}=O\left(\frac{1}{i^{2}}\right)$, we have $c_{m}=O\left(\frac{1}{m+1}\right)$. Using $|D|=1$, we can easily show the following lemma. 
Lemma 4.3.2. Let $c_{m}$ be defined by (4.6). The linear transformation $K: \mathcal{L} \rightarrow \mathcal{L}$ induces a bounded linear transformation $K: \mathcal{L}^{\infty} \rightarrow \mathcal{L}^{\infty}$, whose operator norm $\|K\|$ is estimated as

$$
\|K\| \leq c_{m} .
$$

Lemma 4.3.3. Let $m_{0}$ be the smallest number such that $c_{m_{0}}<1$ (cf. (4.6)). Then,

$$
N_{n m}-D^{n-m}=O\left(\frac{1}{m+1}\right) \quad\left(n \geq m \geq m_{0}\right) .
$$

Proof. Lemma 4.3.2 implies that, for all $m \geq m_{0}, K: \mathcal{L}^{\infty} \rightarrow \mathcal{L}^{\infty}$ is a contraction mapping. By the contraction mapping principle, for any $\Lambda \in \mathcal{L}^{\infty}$, the equation (4.5) has a unique solution $L^{\infty}(\Lambda) \in \mathcal{L}^{\infty}$. Lemma 4.3.1.(1) implies that if $\Lambda \in \mathcal{L}^{\infty}$ then $L^{\infty}(\Lambda)=L(\Lambda)$. Applying this fact to $D^{(m)} \in \mathcal{L}^{\infty}$ and using Lemma 4.3.1(2), we obtain $N^{(m)}=L^{\infty}\left(D^{(m)}\right) \in \mathcal{L}^{\infty}$. Explicitly, $N^{(m)}$ is given by the C. Neumann series:

$$
N^{(m)}=D^{(m)}+\sum_{i=1}^{\infty} K^{i} D^{(m)} .
$$

By Lemma 4.3.2 and $\left\|D^{(m)}\right\|=1$, we have

$$
\begin{aligned}
\left\|N^{(m)}-D^{(m)}\right\| & \leq \sum_{i=1}^{\infty}\|K\|^{i}\left\|D^{(m)}\right\| \leq \sum_{i=1}^{\infty}\left(c_{m}\right)^{i} \\
& \leq \frac{c_{m}}{1-c_{m}}=O\left(\frac{1}{m+1}\right) .
\end{aligned}
$$

This means $\left|N_{n m}-D^{n-m}\right|=O\left(\frac{1}{m+1}\right)\left(n \geq m \geq m_{0}\right)$.

4.4 Technical lemma. We list the technical lemmas that are used later.

Lemma 4.4.1. Let $r$ and $N$ be constants such that $0 \leq \rho<1$ and $N>0$.

$$
\sum_{i=1}^{n} \frac{\rho^{n-i}}{i^{N}}=O\left(\frac{1}{n^{N}}\right) \quad(n \geq 1) .
$$

Proof.

$$
\begin{aligned}
\sum_{i=1}^{n} \frac{\rho^{n-i}}{i^{N}} & \leq \sum_{i=1}^{[n / 2]} \frac{\rho^{n-i}}{i^{N}}+\sum_{i=[n / 2]+1}^{n} \frac{\rho^{n-i}}{i^{N}} \\
& \leq \sum_{i=1}^{[n / 2]} \rho^{n-i}+\frac{1}{([n / 2]+1)^{N}} \sum_{i=[n / 2]+1}^{n} \rho^{n-i} \\
& =\rho^{n-[n / 2]} \frac{1-\rho^{[n / 2]}}{1-\rho}+\frac{1}{([n / 2]+1)^{N}} \frac{1-\rho^{n-[n / 2]}}{1-\rho}=O\left(\frac{1}{n^{N}}\right) .
\end{aligned}
$$

In order to obtain a precise estimate for $N_{n m}$, we use the following lemma. 
Lemma 4.4.2. Let $\rho$ be a constant such that $0 \leq \rho<1$.

$$
\sum_{i=m+1}^{n} \frac{1}{i^{2}}=O\left(\frac{1}{m+1}\right)
$$

$$
\sum_{i=m+1}^{n} \frac{\rho^{n-i}}{i}=O\left(\frac{1}{n+1}\right)
$$

$$
\frac{\rho^{n-m}}{m+1}=O\left(\frac{1}{n+1}\right)
$$

(2) $\sum_{i=m+1}^{n} \frac{\rho^{i-m-1}}{i^{2}}=O\left(\frac{1}{(m+1)^{2}}\right)$,

(4) $\sum_{i=m+1}^{n} \frac{\rho^{n-i}}{i^{2}}=O\left(\frac{1}{(n+1)(m+1)}\right)$,

$(n \geq m \geq 0)$.

Proof. Assertions (1) and (2) are easy; (3) is an immediate consequence of Lemma 4.4.1; (4) easily follows from (3). Finally (5) is shown from the fact that $(n+1) \rho^{n}$ is nonincreasing for $n$ sufficiently large.

4.5. Estimates. Let us obtain an estimate for $N_{n m}$ and then for $M_{n m}$.

Lemma 4.5.1. Let $m_{0}$ be the integer defined in Lemma 4.3.3. Then,

$$
\begin{aligned}
N_{n m}= & \left(\begin{array}{cc}
1+O\left(\frac{1}{m+1}\right) & O\left(\frac{1}{(m+1)^{2}}\right) \\
O\left(\frac{1}{(n+1)(m+1)}\right) & \rho^{n-m}+O\left(\frac{1}{m+1}\left(\rho^{n-m+1}+\frac{1}{(m+1)(n+1)}\right)\right)
\end{array}\right) \\
& \quad\left(n \geq m \geq m_{0}\right) .
\end{aligned}
$$

Proof. Since $N_{m m}=I$ we may assume $n>m \geq m_{0}$. By Lemma 4.3.1(2) we have

$$
N_{n m}=D^{n-m}+\sum_{i=m+1}^{n} D^{n-i} E_{i} N_{i-1, m}
$$

Using Lemma 4.3.3 we can easily show that

$$
D^{n-i} E_{i} N_{i-1, m}=\left(\begin{array}{cc}
O\left(\frac{1}{i^{2}}\right) & O\left(\frac{1}{i^{2}}\left(\rho^{i-m-1}+\frac{1}{m+1}\right)\right) \\
O\left(\frac{\rho^{n-i}}{i^{2}}\right) & O\left(\frac{1}{i^{2}}\left(\rho^{i-m-1}+\frac{\rho^{n-i}}{m+1}\right)\right)
\end{array}\right) \quad\left(n \geq i>m \geq m_{0}\right) .
$$

Applying (1),(2),(4) and (5) in Lemma 4.4.2 to (4.7)-(4.8), we establish the lemma.

\section{Proposition 4.5.2.}

$$
M_{n m}=\left(\begin{array}{cc}
O(1) & O\left(\frac{1}{m+1}\right) \\
O\left(\frac{1}{m+1}\right) & O\left(\rho^{n-m}+\frac{1}{(n+1)(m+1)}\right)
\end{array}\right) \quad(n \geq m \geq 0) .
$$

Proof. Applying Lemma 4.5.1 to $M_{n m}=S_{n} N_{n m}\left(S_{m}\right)^{-1}$, we obtain

$$
M_{n m}=\varepsilon_{m}\left(\begin{array}{cc}
1+O\left(\frac{1}{m+1}\right) & \alpha_{n m}+O\left(\frac{1}{(m+1)^{2}}\right) \\
\beta_{n m}+O\left(\frac{1}{m+1}\left(\frac{1}{n+1}+\frac{\rho^{n-m}}{m+1}\right)\right) & \gamma_{n m}\left(1+O\left(\frac{1}{m+1}\right)\right)
\end{array}\right) \quad\left(n \geq m \geq m_{0}\right),
$$

where $m_{0}$ is defined in Lemma 4.3.3 and

$$
\begin{aligned}
\alpha_{n m} & =\alpha\left(\frac{1}{m+1}-\frac{\rho^{n-m}}{n+1}\right), & \beta_{n m} & =\beta\left(\frac{1}{n+1}-\frac{\rho^{n-m}}{m+1}\right), \\
\gamma_{n m} & =\rho^{n-m}+\frac{\alpha \beta}{(n+1)(m+1)}, & \varepsilon_{m} & =\frac{1}{1+\frac{\alpha \beta}{(m+1)^{2}}} .
\end{aligned}
$$

Using Lemma 4.4.2.(5) we obtain the proposition for $n \geq m \geq m_{0}$. Next we consider the case $0 \leq m<m_{0}$. The subcase $0 \leq m \leq n<m_{0}$ is trivial. In the 
other subcase $n \geq m_{0}>m \geq 0$, we divide $M_{n m}$ as $M_{n m}=M_{n m_{0}} M_{m_{0} m}$. Applying the proposition (for $n \geq m=m_{0}$ ) to $M_{n m_{0}}$, we establish the proposition in this subcase. In conclusion, the proposition holds for all $n \geq m \geq 0$.

4.6. Majorants. Now we are in a position to establish Theorem 4.1.3. We use the method of majorants. For $G=\left(g_{i j}\right), H=\left(h_{i j}\right) \in M_{2}(\mathbb{R})$, we write $G \leq H$ if $g_{i j} \leq h_{i j}$ for all $i, j=1,2$. We fix any $|\cdot| \in \mathcal{N}$. To each $A \in \mathcal{B}$ we associate the following $2 \times 2$-matrix:

$$
\|A\|=\left(\begin{array}{ll}
|X A X| & |X A Y| \\
|Y A X| & |Y A Y|
\end{array}\right)
$$

The following lemma is easy to see.

Lemma 4.6.1.

$$
\|A B\| \leq\|A\|\|B\| \quad(\forall A, B \in \mathcal{B}) .
$$

Now we complete the proof of Theorem 4.1.3. By Assumption A we can choose the constants $a, b>0$ in (4.1) so that

$$
\left\|P_{n}\right\| \leq M_{n} \quad(n \geq 1)
$$

where the matrix $M_{n}$ is given by (4.1). Under Assumption $\mathrm{A}_{u}$ the constants $a, b$ and the minor term $O\left((n+1)^{-2}\right)$ in (4.1) are uniform with respect to $|\cdot| \in \mathcal{N}$. Hence, by Lemma 4.6.1, we have for $n>m \geq 0$,

$$
\left\|P_{n m}\right\| \leq\left\|P_{n}\right\|\left\|P_{n-1}\right\| \cdots\left\|P_{m+1}\right\| \leq M_{n} M_{n-1} \cdots M_{m+1}=M_{n m} .
$$

This is also valid for $n=m$. Proposition 4.5.2 implies Theorem 4.1.3.

4.7. Limit propagators. As a collorary to Theorem 4.1.3 we obtain the following theorem.

Theorem 4.7.1. Under Assumption A there exists an infinite sequence $\left\{R_{m}\right\}$ of operators in $\mathcal{B}$ such that

$$
\begin{array}{ll}
X R_{m}=R_{m}, & \\
R_{m} X-X P_{n m} X=O\left(\frac{1}{n+1}\right) & (n \geq m \geq 0), \\
R_{m} Y-X P_{n m} Y=O\left(\frac{1}{n+1}\left(\rho^{n-m}+\frac{1}{m+1}\right)\right) & (n \geq m \geq 0) .
\end{array}
$$

Under Assumption $\mathrm{A}_{u}$ the Landau symbol $O$ can be replaced by $O_{u}$.

Proof. Theorem 4.1.3 implies that, for any fixed $m,\left\{X P_{n m}\right\}_{n \geq m}$ is a Cauchy sequence in $\mathcal{B}$. Since $\mathcal{B}$ is sequentially complete (cf. Remark 3.1.1), there exists an $R_{m} \in \mathcal{B}$ such that $X P_{n m} \rightarrow R_{m}$ in $\mathcal{B}$ as $n \rightarrow \infty$. Then Assertion (1) is clear. Letting $k \rightarrow \infty$ in Theorem 4.1.3, we also obtain (2) and (3).

Putting $n=m$ in (2)(3) of Theorem 4.7.1, we obtain the following corollary.

Corollary 4.7.2. Under Assumption A,

$$
R_{m} X=X+O\left(\frac{1}{m+1}\right), \quad R_{m} Y=O\left(\frac{1}{m+1}\right) \quad(m \geq 0) .
$$

Under Assumption $\mathrm{A}_{u}$ the Landau symbol $O$ can be replaced by $O_{u}$.

Definition 4.7.3. The operator $R_{m}$ in Theorem 4.7 .1 is called the limit propagator (from time $m$ ). 
4.8. Profile operator. Now we are in a position to define the profile operator $\Phi$.

Definition 4.8.1. For $n \geq 0$ we define a bounded linear operator $\Phi_{n}: \ell^{0} \rightarrow U_{1}$ by

$$
\Phi_{n} v=\sum_{m=n}^{\infty} R_{m} v_{m} \quad\left(v=\left(v_{n}\right) \in \ell^{0}\right) .
$$

Lemma 4.8.2 below shows that $\Phi_{n}$ is well-defined. We set $\Phi=\Phi_{0}$ and call it the profile operator.

Lemma 4.8.2. Under Assumption $\mathrm{A}, \Phi_{n}$ is well-defined, i.e., the infinite series in (4.9) is (absolutely) convergent and $\Phi_{n} v$ admits the following estimate:

$$
\left|\Phi_{n} v\right|=O\left(\frac{|v|_{0}}{n+1}\right) \quad\left(v \in \ell^{0}, n \geq 0\right) .
$$

Under Assumption $\mathrm{A}_{u}$ the Landau symbol $O$ can be replaced by $O_{u}$.

Proof. We only consider the case of Assumption A. In the case of Assumption $\mathrm{A}_{u}$ we have only to replace $O$ by $O_{u}$ in the proof. Corollary 4.7.2 implies

$$
\begin{aligned}
\left|R_{m} v_{m}\right| & \leq\left|R_{m} X\right|\left|X v_{m}\right|+\left|R_{m} Y\right|\left|Y v_{m}\right| \\
& =O\left(\frac{1}{m+1}(m+1)\left|X_{m}\right|+\frac{1}{(m+1)^{2}} \sup _{k \geq 0}(k+1)\left|v_{k}\right|\right) \quad(m \geq 0),
\end{aligned}
$$

and hence

$$
\begin{aligned}
\sum_{m=n}^{k}\left|R_{m} v_{m}\right| & =O\left(\sum_{m=n}^{k} \frac{1}{m+1}(m+1)\left|X v_{m}\right|+\sup _{k \geq 0}(k+1)\left|v_{k}\right| \sum_{m=n}^{k} \frac{1}{(m+1)^{2}}\right) \\
& =O\left(\frac{1}{n+1}\left\{\sum_{m=n}^{\infty}(m+1)\left|X_{m}\right|+\sup _{k \geq 0}(k+1)\left|v_{k}\right|\right\}\right) \\
& =O\left(\frac{|v|_{0}}{n+1}\right) \quad(k \geq n \geq 0) .
\end{aligned}
$$

Therefore the sequential completeness of $U$ implies that (4.9) is converegent.

Now we can state the main theorem in this section.

Theorem 4.8.3 (Theorem I). (1) Under Assumption A there exists a bounded linear operator $\Phi: \ell^{0} \rightarrow U_{1}$ (the profile operator) such that

$$
\left|\phi_{n}(v)-\Phi v\right|=O\left(\frac{|v|_{0}}{n+1}\right) \quad\left(v \in \ell^{0}, n \geq 0\right) .
$$

(2) Under Assumption $\mathrm{A}_{u}$ the Landau symbol $O$ can be replaced by $O_{u}$. Moreover there exists an integer $m_{0}$ such that $\Phi \circ \iota_{m}: U_{1} \rightarrow U_{1}$ is a topological isomorphism for all $m \geq m_{0}$, where $\iota_{m}$ is defined in Definition 3.2.3.

Proof. By Lemma 4.1.2 and Definition 4.8.1, we have $\Phi v-\phi_{n}=J+\Phi_{n+1} v$, where

$$
J=\sum_{m=0}^{n}\left(R_{m}-P_{n m}\right) v_{m}
$$


By using Theorem 4.1.3 and Theorem 4.7.1, $J$ is estimated as follows:

$$
\begin{aligned}
|J| \leq & \sum_{m=0}^{n}\left(\left|R_{m} X-X P_{n m} X\right|+\left|Y P_{n m} X\right|\right)\left|X v_{m}\right| \\
& +\sum_{m=0}^{n}\left|R_{m} Y-X P_{n m} Y\right|\left|Y v_{m}\right|+\sum_{m=0}^{n}\left|Y P_{n m} Y\right|\left|Y v_{m}\right| \\
\leq & O\left(\frac{1}{n+1} \sum_{m=0}^{n}\left|X v_{m}\right|+\frac{1}{n+1} \sum_{m=0}^{n}\left\{\rho^{n-m}+\frac{1}{m+1}\right\}\left|Y v_{m}\right|\right) \\
& +O\left(\sum_{m=0}^{n}\left\{\rho^{n-m}+\frac{1}{(n+1)(m+1)}\right\}\left|Y v_{m}\right|\right) \\
= & O\left(\frac{|v|_{0}}{n+1}+\sum_{m=0}^{n}\left\{\rho^{n-m}+\frac{1}{(n+1)(m+1)}\right\}\left|Y v_{m}\right|\right) \\
= & O\left(\frac{|v|_{0}}{n+1}+\left\{\sum_{m=0}^{n} \frac{\rho^{n-m}}{m+1}+\frac{1}{n+1} \sum_{m=0}^{n} \frac{1}{(m+1)^{2}}\right\}|v|_{0}\right) \\
= & O\left(\frac{|v|_{0}}{n+1}\right) .
\end{aligned}
$$

Here Lemma 4.4.1 $(N=1)$ is used in the last equality. This estimate and Lemma 4.8.2 establish (1) of the theorem. The first assertion of (2) is established in a similar manner.

It remains to establish the second assertion of (2). We set $\Phi \circ \iota_{m}=I_{1}-F_{m}$. Definition 4.8.1 implies $\Phi \circ \iota_{m}=\left.R_{m}\right|_{U_{1}}$. By Corollary 4.7.2 we have $\left.R_{m}\right|_{U_{1}}=$ $I_{1}+O_{u}\left(\frac{1}{m+1}\right)$. So there exist $m_{0} \geq 0$ and $\rho_{1}\left(0 \leq \rho_{1}<1\right)$ such that $\left|F_{m}\right| \leq \rho_{1}$ for any $m \geq m_{0}$ and $|\cdot| \in \mathcal{N}$. By the sequential completeness of $\mathcal{B}$, the C. Neumann series $\sum_{i=0}^{\infty}\left(F_{m}\right)^{i}$ converges in $\mathcal{B}$ and gives the inverse of $\Phi \circ \iota_{m}$. Hence, for any $m \geq m_{0}, \Phi \circ \iota_{m}$ is a linear isomorphism.

Remark 4.8.4. The second assertion of (2) in Theorem 4.8.3 was established by using the estimate

$$
\left.R_{m}\right|_{U_{1}}=I_{1}+O_{u}\left(\frac{1}{m+1}\right) .
$$

If $O_{u}$ is replaced by $O$ in (4.10), then it is not always possible to take $m_{0}$ and $\rho_{1}$ uniformly with respect to $|\cdot| \in \mathcal{N}$. Hence the C. Neumann series $\sum_{i=0}^{\infty}\left(F_{m}\right)^{i}$ is not necessarily convergent in $\mathcal{B}$. Our argument fails at this point. Assumption $\mathrm{A}_{u}$ leads to the estimate (4.10), while Assumption A leads only to the estimate (4.10) with $O_{u}$ replaced by $O$. For this reason we need Assumption $\mathrm{A}_{u}$ in place of Assumption A.

\section{Asymptotic Expansions}

5.1. Induction. We establish the following theorem by induction.

Theorem 5.1.1 (Assertion (1) of Theorem II). Under Assumption $\mathrm{B}_{N}$,

$$
\left|\phi_{n}(v)-\left(\sum_{i=0}^{N} \Psi_{i}[n]_{i}\right) \Phi v\right|=O\left(\frac{|v|_{N}}{(n+1)^{N+1}}\right) \quad\left(v \in \ell^{N}, n \geq N\right) .
$$


Assertion (2) of Theorem II is an immediate consequence of Theorem 5.1.1, because Assumption $\mathrm{B}_{\infty}$ implies Assumption $\mathrm{B}_{N}$ for any $N \in \mathbb{Z}_{>0}$. In Assumption $\mathrm{B}_{N}$, if $N=0$ then Theorem 5.1.1 immediately follows from Theorem I (under Assumption A). In this section we assume $N \geq 1$. We use the following notation.

Notation 5.1.2. For $m=0,1, \ldots, N$ and $1 \leq j+k \leq m+1$, we set

$$
\begin{aligned}
F_{m}(n) v & =\phi_{n}(v)-\left(\sum_{i=0}^{m} \Psi_{i}[n]_{i}\right) \Phi v, \\
E_{m}(n) & =P_{n}-\sum_{i=0}^{m+1} P^{(i)}(n)_{i}, \\
\varepsilon_{j k m}(n) & =(n)_{k}[n-1]_{j}-\sum_{i=j+k}^{m+1}[j, k]\left[\begin{array}{c}
k-1 \\
i-j-k
\end{array}\right][n]_{i},
\end{aligned}
$$

(cf. Definitions 2.2.1, 3.2.6 and Notation 2.2.2).

In order to set up an induction argument, we consider the following claims.

Claims 5.1.3. For any fixed $|\cdot| \in \mathcal{N}$,

$$
\begin{aligned}
\left|Y F_{m}(n) v\right|=O\left(\frac{|v|_{m}}{(n+1)^{m+1}}\right) & \left(v \in \ell^{m}, n \geq m\right), \\
\left|X F_{m}(n) v\right|=O\left(\frac{|v|_{m}}{(n+1)^{m+1}}\right) & \left(v \in \ell^{m}, n \geq m\right), \\
\left|F_{m}(n) v\right|=O\left(\frac{|v|_{m}}{(n+1)^{m+1}}\right) & \left(v \in \ell^{m}, n \geq m\right) .
\end{aligned}
$$

First we note that Theorem I (under Assumption A) implies (5.3.0), and hence (5.1.0) and (5.2.0). Under Assumption $\mathrm{B}_{N}$ these claims make sense for $m=$ $1, \ldots, N$ (of course, it is a priori not clear whether they are true or not). The induction argument proceeds through the following three steps.

Step 1. (5.3.m) implies $(5.1 . m+1)$ for $m=0,1, \ldots, N-1$.

Step 2. $(5.3 . m)$ and $(5.1 . m+1)$ imply $(5.2 . m+1)$ for $m=0,1, \ldots, N-1$.

Step 3. (5.1.m) and (5.2.m) imply (5.3.m) for $m=0,1, \ldots, N$.

We establish Step 1 and Step 2; Step 3 is trivial. Then Theorem 5.1.1 follows from $(5.3 \cdot N)$.

5.2. Technical lemmas. In what follows we use the following lemmas.

Lemma 5.2.1. Let $0 \leq \rho<1$ and $N \in \mathbb{Z}_{\geq 0}$. If $\left\{a_{n}\right\}_{n \geq 0}$ is an infinite sequence of nonnegative numbers such that

$$
a_{n}-\rho a_{n-1}=O\left(\frac{1}{n^{N}}\right) \quad(n \geq 1),
$$

then

$$
a_{n}=O\left(\frac{1}{(n+1)^{N}}\right) \quad(n \geq 0) .
$$


Proof. We set $b_{n}=\rho^{-n} a_{n}$. Then we have $b_{i}-b_{i-1}=O\left(\rho^{-i} i^{-N}\right)$. Summing it over $i=1,2, \ldots, n$, we have $b_{n}-b_{0}=O\left(\sum_{i=1}^{n} \rho^{-i} i^{-N}\right)$. Hence, using Lemma 4.4.1, we obtain

$$
a_{n}=a_{0} \rho^{n}+O\left(\sum_{k=1}^{n} \frac{\rho^{n-k}}{k^{N}}\right)=O\left(\frac{1}{(n+1)^{N}}\right)
$$

Lemma 5.2.2. For $m=0,1, \ldots, N$ and $1 \leq j+k \leq m+1$,

$$
E_{m}(n)=O\left(\frac{1}{n^{m+2}}\right), \quad \varepsilon_{j k m}(n)=O\left(\frac{1}{n^{m+2}}\right) \quad(n \geq m+1) .
$$

This lemma is an immediate consequence of Lemma 2.2.5 and Assumption $\mathrm{B}_{N}$.

\subsection{Step 1.}

Lemma 5.3.1. If (5.3.m) holds, then we have

$$
\left(Y P_{n}-Z\right) \phi_{n-1}=\sum_{i=1}^{m+1} \sum_{j=0}^{i-1} Y P_{i j} \Psi_{j}[n]_{i} \Phi v+\Delta_{m}(n) v
$$

with

$$
\left|\Delta_{m}(n) v\right|=O\left(\frac{|v|_{m}}{n^{m+2}}\right) \quad\left(v \in \ell^{m}, n \geq m+1\right)
$$

Proof. By (1) of Assumption $\mathrm{B}_{N}$ we have

$$
Y P_{n}-Z=\sum_{i=1}^{m+1} Y P^{(i)}(n)_{i}+Y E_{m}(n) .
$$

Here the point is that the suffix $i$ starts from $i=1$. By Notation 5.1.2 we have

$$
\left(Y P_{n}-Z\right) \phi_{n-1}=\sum_{\substack{0 \leq j \leq m \\ 1 \leq k \leq m+1 \\ j+k \leq m+1}} Y P^{(k)} \Psi_{j}(n)_{k}[n-1]_{j} \Phi v+\Theta_{m}(n) v
$$

where

$$
\begin{aligned}
\Theta_{m}(n) & =\sum_{i=1}^{m+1} Y P^{(i)}(n)_{i} F_{m}(n-1)+Y E_{m}(n) \sum_{j=0}^{m} \Psi_{j}[n-1]_{j} \Phi \\
& +Y E_{m}(n) \cdot F_{m}(n-1)+\sum_{\substack{1 \leq i \leq m+1 \\
0 \leq j \leq m \\
i+j \geq m+2}} Y P^{(i)} \Psi_{j}(n)_{i}[n-1]_{j} \Phi .
\end{aligned}
$$


Moreover, by Notation 5.1.2, we have

$$
\begin{aligned}
\left(Y P_{n}-Z\right) \phi_{n-1} & \\
= & \sum_{\substack{0 \leq j \leq m \\
1 \leq k \leq m+1 \\
j+k \leq m+1}} Y P^{(k)} \Psi_{j}\left(\sum_{i=j+k}^{m+1}[j, k]\left[\begin{array}{c}
k-1 \\
i-j-k
\end{array}\right][n]_{i}+\varepsilon_{j k m}(n)\right) \Phi v+\Theta_{m}(n) v \\
= & \sum_{i=1}^{m+1} \sum_{j=0}^{i-1} Y \sum_{k=1}^{i-j}[j, k]\left[\begin{array}{c}
k-1 \\
i-j-k
\end{array}\right] P^{(k)} \Psi_{j}[n]_{i} \Phi v+\Delta_{m}(n) v \\
= & \sum_{i=1}^{m+1} \sum_{j=0}^{i-1} Y P_{i j} \Psi_{j}[n]_{i} \Phi v+\Delta_{m}(n) v
\end{aligned}
$$

where

$$
\Delta_{m}(n)=\sum_{\substack{0 \leq j \leq m \\ 1 \leq k \leq m+1 \\ j \neq k \leq m+1}} Y P^{(k)} \Psi_{j} \Phi \varepsilon_{j k m}(n)+\Theta_{m}(n) .
$$

Applying (5.3.m) and Lemma 5.2.2 to $\Delta_{m}(n) v$, we obtain the desired estimate.

Proof of Step 1. By using the difference equation (1.8) and Lemma 2.2.3, we have

$$
\begin{aligned}
Y F_{m+1}(n) v & -Z Y F_{m+1}(n-1) v \\
& =Y \phi_{n}-Z Y \phi_{n-1}-\sum_{i=1}^{m+1}\left(Y \Psi_{i}[n]_{i}-Z Y \Psi_{i}[n-1]_{i}\right) \Phi v \\
& =\left(Y P_{n}-Z\right) \phi_{n-1}+Y v_{n}-\sum_{i=1}^{m+1}\left\{\left(I_{0}-Z\right) Y \Psi_{i}[n]_{i}+Z \Psi_{i}[n]_{i+1}\right\} \Phi v \\
& =\left(Y P_{n}-Z\right) \phi_{n-1}-\sum_{i=1}^{m+1}\left\{\left(I_{0}-Z\right) Y \Psi_{i}+Z \Psi_{i-1}\right\}[n]_{i} \Phi v \\
& +Y v_{n}-Z \Psi_{m+1}[n]_{m+2} \Phi v .
\end{aligned}
$$

By Notation 3.2.5 and Definition 3.2.6, we have

$$
\begin{aligned}
Y F_{m+1}(n) v & -Z Y F_{m+1}(n-1) v \\
& =\sum_{i=1}^{m+1}\left\{-\left(I_{0}-Z\right) Y \Psi_{i}+\sum_{j=0}^{i-1}\left(Y P_{i j}-\delta_{i, j+1} Z\right) \Psi_{j}\right\}[n]_{i} \Phi v+\Xi_{m}(n) v, \\
& =\sum_{i=1}^{m+1}\left(I_{0}-Z\right) Y\left(-\Psi_{i}+\sum_{j=0}^{i-1} A_{i j} \Psi_{j}\right)[n]_{i} \Phi v+\Xi_{m}(n) v=\Xi_{m}(n) v,
\end{aligned}
$$

where

$$
\Xi_{m}(n) v=Y v_{n}-Z \Psi_{m+1}[n]_{m+2} \Phi v+\Delta_{m}(n) v .
$$

Lemma 5.3.1 implies

$$
\left|\Xi_{m}(n) v\right|=O\left(\frac{|v|_{m+1}}{(n+1)^{m+2}}\right) \quad\left(v \in \ell^{m+1}, n \geq m+1\right),
$$


and hence

$$
\begin{aligned}
\left|Y F_{m+1}(n) v\right| & \leq|Z|\left|Y F_{m+1}(n-1) v\right|+\left|\Xi_{m}(n) v\right| \\
& \leq r\left|Y F_{m+1}(n-1) v\right|+O\left(\frac{|v|_{m+1}}{(n+1)^{m+2}}\right) .
\end{aligned}
$$

Finally, applying Lemma 5.2.1 to this inequality, we establish Step 1.

\subsection{Step 2.}

Lemma 5.4.1. If (5.3.m) and (5.1.m+1) hold, then we have

$$
X \phi_{n}-X \phi_{n-1}=\sum_{i=1}^{m+1} X \Psi_{i}[n]_{i+1} \Phi v+\Delta_{m}(n) v,
$$

with

$$
\left|\Delta_{m}(n) v\right|=O\left(\frac{|v|_{m+1}}{(n+1)^{m+3}}\right) \quad\left(v \in \ell^{m+1}, n \geq m+2\right) .
$$

Proof. By Assumption $\mathrm{B}_{N}$ and Notation 5.1.2, we have

$$
\begin{aligned}
X P_{n} X-X & =\sum_{i=2}^{m+2} X P^{(i)} X(n)_{i}+X E_{m+1}(n) X, \\
X P_{n} Y & =\sum_{i=1}^{m+1} X P^{(i)} Y(n)_{i}+X E_{m}(n) Y .
\end{aligned}
$$

Here the point is that the suffix $i$ in the summation in (5.4) (resp. (5.5)) starts from $i=2$ (resp. $i=1$ ). By Notation 5.1.2 and $Y \Psi_{0}=Y X=O$, we have

$$
\begin{aligned}
\phi_{n-1} & =\sum_{j=0}^{m} \Psi_{j}[n-1]_{j} \Phi v+F_{m}(n-1) v, \\
Y \phi_{n-1} & =\sum_{j=1}^{m+1} Y \Psi_{j}[n-1]_{j} \Phi v+Y F_{m+1}(n-1) v .
\end{aligned}
$$

The difference equation (1.8) yields

$$
X \phi_{n}-X \phi_{n-1}=\left(X P_{n} X-X\right) \phi_{n-1}+X P_{n} Y \cdot Y \phi_{n-1}+X v_{n} .
$$

Substituting the four equalities (5.4)-(5.7) into (5.8), we obtain

$$
\begin{aligned}
X \phi_{n}-X \phi_{n-1} & =\sum_{\substack{0 \leq j \leq m \\
2 \leq k \leq m+2 \\
j+k \leq m+2}} X P^{(k)} X \Psi_{j}(n)_{k}[n-1]_{j} \Phi v \\
& +\sum_{\substack{1 \leq j \leq m+1 \\
1 \leq k \leq m+1 \\
j+k \leq m+2}} X P^{(k)} Y \Psi_{j}(n)_{k}[n-1]_{j} \Phi v+\Theta_{m}(n) v,
\end{aligned}
$$


where

$$
\begin{aligned}
\Theta_{m}(n) & =\sum_{\substack{2 \leq i \leq m+2 \\
0 \leq j \leq m \\
i+j \geq m+3}} X P^{(i)} X \Psi_{j}(n)_{i}[n-1]_{j} \Phi+X E_{m+1}(n) X \sum_{j=0}^{m} \Psi_{j}[n-1]_{j} \Phi \\
& +\sum_{i=2}^{m+2} X P^{(i)} X(n)_{i} \cdot F_{m}(n-1)+X E_{m+1}(n) X \cdot F_{m}(n-1) \\
& +\sum_{\substack{1 \leq i \leq m+1 \\
1 \leq j \leq m+1 \\
i+j \leq m+3}} X P^{(i)} Y \Psi_{j}(n)_{i}[n-1]_{j} \Phi+X E_{m}(n) Y \sum_{j=1}^{m+1} \Psi_{j}[n-1]_{j} \Phi \\
& +\sum_{i=1}^{m+1} X P^{(i)}(n)_{i} \cdot Y F_{m+1}(n-1)+X E_{m}(n) \cdot Y F_{m+1}(n-1) \\
& +X v_{n} .
\end{aligned}
$$

Moreover, using Notation 5.1.2, we have

$$
\begin{aligned}
X \phi_{n}- & X \phi_{n-1} \\
= & \sum_{\substack{0 \leq j \leq m \\
2 \leq k \leq m+2 \\
j+k \leq m+2}} X P^{(k)} X \Psi_{j}\left(\sum_{i=j+k}^{m+2}[j, k]\left[\begin{array}{c}
k-1 \\
i-j-k
\end{array}\right][n]_{i}+\varepsilon_{j k, m+1}(n)\right) \Phi v \\
& +\sum_{\substack{1 \leq j \leq m+1 \\
1 \leq k \leq m+1 \\
j+k \leq m+2}} X P^{(k)} Y \Psi_{j}\left(\sum_{i=j+k}^{m+2}[j, k]\left[\begin{array}{c}
k-1 \\
i-j-k
\end{array}\right][n]_{i}+\varepsilon_{j k, m+1}(n)\right) \Phi v \\
& +\Theta_{m}(n) v \\
& =\sum_{i=2}^{m+2} \sum_{j=0}^{i-2} X\left(\sum_{k=2}^{i-j}[j, k]\left[\begin{array}{c}
k-1 \\
i-j-k
\end{array}\right] P^{(k)}\right) X \Psi_{j}[n]_{i} \Phi v \\
& +\sum_{i=2}^{m+2} \sum_{j=1}^{i-1} X\left(\sum_{k=1}^{i-j}[j, k]\left[\begin{array}{c}
k-1 \\
i-j-k
\end{array}\right] P^{(k)}\right) Y \Psi_{j}[n]_{i} \Phi v+\Delta_{m}(n) v,
\end{aligned}
$$

where

$$
\begin{aligned}
\Delta_{m}(n)=\Theta_{m}(n)+ & \sum_{\substack{0 \leq j \leq m \\
2 \leq k \leq m+2 \\
j+k \leq m+2}} X P^{(k)} X \Psi_{j} \Phi \varepsilon_{j k, m+1}(n) \\
& +\sum_{\substack{1 \leq j \leq m+1 \\
1 \leq k \leq m+1 \\
j+k \leq m+2}} X P^{(k)} Y \Psi_{j} \Phi \varepsilon_{j k, m+1}(n) .
\end{aligned}
$$


Using Notation 3.2.4, we have

$$
\begin{aligned}
X \phi_{n}-X \phi_{n-1} & =\sum_{i=2}^{m+2} \sum_{j=0}^{i-2} X P_{i j} \Psi_{j}[n]_{i} \Phi v \\
& +\sum_{i=2}^{m+2}[i-1,1] X P^{(1)} Y \Psi_{i-1}[n]_{i} \Phi v+\Delta_{m}(n) v \\
& =\sum_{i=1}^{m+1}\left(\sum_{j=0}^{i-1} X P_{i+1, j} \Psi_{j}+\frac{1}{i} X P^{(1)} Y \Psi_{i}\right)[n]_{i+1} \Phi v+\Delta_{m}(n) v
\end{aligned}
$$

By Notation 3.2.5 and Definition 3.2.6, we have

$$
\begin{aligned}
\sum_{j=0}^{i-1} X P_{i+1, j} \Psi_{j} & +\frac{1}{i} X P^{(1)} Y \Psi_{i} \\
& =\sum_{j=0}^{i-1}\left(X P_{i+1, j}+\frac{1}{i} X P^{(1)} Y A_{i j}\right) \Psi_{j} \\
& =\sum_{j=0}^{i-1}\left(X P_{i+1, j}+\frac{1}{i} X P^{(1)}\left(I_{0}-Z\right)^{-1}\left(Y P_{i j}-\delta_{i, j+1} Z\right)\right) \Psi_{j} \\
& =\sum_{j=0}^{i-1} X A_{i j} \Psi_{j}=X \Psi_{i} .
\end{aligned}
$$

Hence we obtain

$$
X \phi_{n}-X \phi_{n-1}=\sum_{i=1}^{m+1} X \Psi_{i}[n]_{i+1} \Phi v+\Delta_{m}(n) v .
$$

The definition of $\Delta_{m}(n)$, Lemma 5.2.2, $(5.3 . m)$ and $(5.1 . m+1)$ yield

$$
\left|\Delta_{m}(n) v\right|=O\left(\frac{|v|_{m+1}}{(n+1)^{m+3}}\right) \quad\left(v \in \ell^{m+1}, n \geq m+2\right) .
$$

Hence the lemma is established.

Proof of Step 2. By Lemma 2.2.3 and Lemma 5.4.1, we have

$$
\begin{aligned}
X F_{m+1}(n) v-X F_{m+1}(n-1) v & =X \phi_{n}-X \phi_{n-1}-\sum_{i=1}^{m+1} X \Psi_{i}\left([n]_{i}-[n-1]_{i}\right) \Phi v \\
& =\sum_{i=1}^{m+1} X \Psi_{i}[n]_{i+1} \Phi v+\Delta_{m}(n) v-\sum_{i=1}^{m+1} X \Psi_{i}[n]_{i+1} \Phi v \\
& =\Delta_{m}(n) v .
\end{aligned}
$$


By Lemma 5.4.1 we have

$$
\begin{aligned}
\mid X F_{m+1}(M) v & -X F_{m+1}(n) v\left|\leq \sum_{k=n+1}^{M}\right| X F_{m+1}(k) v-X F_{m+1}(k-1) v \mid \\
& =\sum_{k=n+1}^{M}\left|\Delta_{m}(k) v\right|=O\left(\sum_{k=n+1}^{M} \frac{|v|_{m+1}}{(k+1)^{m+3}}\right) \\
& =O\left(\frac{|v|_{m+1}}{(n+1)^{m+2}}\right) \quad\left(v \in \ell^{m+1}, M>n \geq m+1\right) .
\end{aligned}
$$

Theorem I implies $F_{m+1}(M) v \rightarrow 0$ as $M \rightarrow \infty$. Letting $M \rightarrow \infty$ in the above formula, we obtain

$$
\left|X F_{m+1}(n) v\right|=O\left(\frac{|v|_{m+1}}{(n+1)^{m+2}}\right) \quad\left(v \in \ell^{m+1}, n \geq m+1\right) .
$$

This establishes Step 2.

5.5. Proof of Corollary 3.4.2. To establish Corollary 3.4 .2 we show that the pair $\left(\ell^{\infty}, P\right)$ satisfies Conditions (AP-1)-(AP-4) in Definition 1.1. Assumption $\mathrm{B}_{\infty}$ and Definition 3.2.2 imply that $P$ and $T$ map $\ell^{\infty}$ into itself, and hence (AP-1). Condition (AP-2) is trivial from Definition 3.2.2. Assertion (2) of Theorem I implies (3) of (AP-3). The other conditions in (AP-3) and (AP-4) readily follow from (2) of Theorem II. Hence $\left(\ell^{\infty}, P\right)$ is an asymptotic pair of order infinity.

\section{Gevrey Estimates}

6.1. Preliminaries. Let $p, q, r$ and $s$ be as in Assumption $\mathrm{C}$ and Notation 3.5.4.

Notation 6.1.1. Let $|\cdot|$ be any semi-norm in $\mathcal{N}$, which is fixed throughout this section. Let $C=\left(C_{1}, C_{2}, C_{3}, C_{4}\right)$ be any constants such that $C_{i}>c_{i}(|\cdot|)(i=$ $1,2,3,4)$, where the $c_{i}(|\cdot|)$ are defined in Definition 3.5.5.

Then Definition 3.5.5 immediately implies the following lemma.

Lemma 6.1.2. For any constants $C$ as in Notation 6.1.1, there exists a nonegative integer $n_{0}(C)$ such that

$$
\begin{aligned}
& \left|X P_{n} X-X\right| \leq \frac{C_{1}}{n(n+1)}, \quad\left|X P_{n} Y\right| \leq \frac{C_{2}}{n^{p}}, \\
& \left|Y P_{n} X\right| \leq \frac{C_{3}}{n^{q}}, \quad\left|Y P_{n} Y\right| \leq \frac{C_{4}}{n^{r}} \quad\left(n \geq n_{0}(C)\right) .
\end{aligned}
$$

Definition 6.1.3. For any $v \in \ell^{1}$ we set

$$
\begin{gathered}
f_{n}(v)=\sum_{k=n}^{\infty} \frac{\left|X \phi_{k}(v)\right|}{(k+1)(k+2)}, \quad g_{n}(v)=\sum_{k=n}^{\infty} \frac{\left|Y \phi_{k}(v)\right|}{(k+1)^{p}}, \\
\alpha_{n}(v)=\sum_{k=n}^{\infty}\left|X v_{k}\right|, \quad \beta_{n}(v)=\sum_{k=n}^{\infty}\left|Y v_{k}\right|, \quad \gamma_{n}(v)=\sum_{k=n}^{\infty}\left|v_{k}\right| .
\end{gathered}
$$

These are often abbreviated as $f_{n}, g_{n}, \alpha_{n}, \beta_{n}, \gamma_{n}$ with $v$ being understood. By Definition 3.2.2 and Theorem II, these infinite series converge and define finite numbers.

Notation 6.1.4. $\ell_{0}^{1}=\left\{v \in \ell^{1} ; \Phi v=0\right\}$. 
6.2. Estimate of $f_{n}$. The following lemma is easily established.

Lemma 6.2.1. For any $\varepsilon>0$ we set $n_{1}(C, \varepsilon)=\max \left\{\left[C_{1}+1 / \varepsilon\right], n_{0}(C)\right\}$, where $[\cdot]$ is the Gauss symbol. Then we have

$$
0<\left(1-\frac{C_{1}}{n+1}\right)^{-1} \leq 1+\varepsilon C_{1} \quad\left(n \geq n_{1}(C, \varepsilon)\right) .
$$

Lemma 6.2.2. Let $\ell_{0}^{\infty}$ be as in Notation 6.1.4. For any $\varepsilon>0$,

$$
f_{n}(v) \leq \frac{1+\varepsilon C_{1}}{n+1}\left\{C_{2} g_{n}(v)+\alpha_{n+1}(v)\right\} \quad\left(n \geq n_{1}(C, \varepsilon), v \in \ell_{0}^{1}\right) .
$$

Proof. The difference equation (1.8) yields, for $k \geq 0$,

$$
\left|X \phi_{k+1}(v)-X \phi_{k}(v)\right| \leq\left|X P_{k+1} X-X\right|\left|X \phi_{k}(v)\right|+\left|X P_{k+1} Y\right|\left|Y \phi_{k}(v)\right|+\left|X v_{k+1}\right| \text {. }
$$

Applying Lemma 6.1.2, we have, for $k \geq n_{0}(C)$,

$$
\left|X \phi_{k+1}(v)-X \phi_{k}(v)\right| \leq C_{1} \frac{\left|X \phi_{k}(v)\right|}{(k+1)(k+2)}+C_{2} \frac{\left|Y \phi_{k}(v)\right|}{(k+1)^{p}}+\left|X v_{k+1}\right| .
$$

Summing over $k=n, n+1, n+2, \ldots$ and taking $\lim _{k \rightarrow \infty} \phi_{k}(v)=0$ into account, we obtain

$$
\left|X \phi_{n}(v)\right| \leq C_{1} f_{n}+C_{2} g_{n}+\alpha_{n+1} \quad\left(n \geq n_{0}(C)\right) .
$$

Since $f_{k}, g_{k}$ and $\alpha_{k}$ are nonincreasing, we have

$$
\frac{\left|X \phi_{k}(v)\right|}{(k+1)(k+2)} \leq \frac{C_{1} f_{k}+C_{2} g_{k}+\alpha_{k+1}}{(k+1)(k+2)} \leq \frac{C_{1} f_{n}+C_{2} g_{n}+\alpha_{n+1}}{(k+1)(k+2)} \quad\left(k \geq n \geq n_{0}(C)\right) .
$$

Summing over $k=n, n+1, n+2, \ldots$, we have

$$
f_{n} \leq \frac{C_{1} f_{n}+C_{2} g_{n}+\alpha_{n+1}}{n+1} \quad\left(n \geq n_{0}(C)\right) .
$$

Using Lemma 6.2.1, we obtain

$$
f_{n} \leq \frac{1+\varepsilon C_{1}}{n+1}\left(C_{2} g_{n}+\alpha_{n+1}\right) \quad\left(n \geq n_{1}(C, \varepsilon)\right) .
$$

\subsection{Estimate of $g_{n}$.}

Definition 6.3.1. We set

$$
C_{0}=\left\{\begin{array}{ll}
C_{2} C_{3} & (p+q-1<r), \\
C_{2} C_{3}+C_{4} & (p+q-1=r), \\
C_{4} & (p+q-1>r),
\end{array} \quad B_{0}=\sqrt{2} \max \left\{C_{3}, 1\right\} .\right.
$$

Lemma 6.3.2. For any $A>C_{0}$ and $B>B_{0}$, there exists a positive constant $\varepsilon=\varepsilon(A, B, C)$ depending only on $(A, B, C)$ such that

$$
\begin{aligned}
\frac{\left(1+\varepsilon C_{1}\right) C_{2} C_{3}}{(n+1)^{p+q-1}}+\frac{C_{4}}{(n+1)^{r}} & \leq \frac{A}{(n+1)^{s}}, \\
\sqrt{2} \max \left\{\left(1+\varepsilon C_{1}\right) C_{3}, 1\right\} & \leq B \quad(n \geq m(A, B, C)),
\end{aligned}
$$

where $m(A, B, C)=n_{1}(C, \varepsilon(A, B, C))$ and $n_{1}(C, \varepsilon)$ is defined in Lemma 6.2.1.

Proof. The lemma easily follows from Definition 6.3.1 and the fact that $n_{1}(C, \varepsilon) \rightarrow$ $\infty$ as $\varepsilon \rightarrow 0$ (cf. Lemma 6.2.1). 
Lemma 6.3.3. For any $A>C_{0}$ and $B>B_{0}$,

$$
g_{n+1}(v) \leq \frac{A g_{n}(v)}{(n+1)^{s}}+B \gamma_{n+1}(v) \quad\left(n \geq m(A, B, C), v \in \ell_{0}^{1}\right),
$$

Proof. The difference equation (1.8) yields

$$
\left|Y \phi_{k+1}(v)\right| \leq\left|Y P_{k+1} X\right|\left|X \phi_{k}(v)\right|+\left|Y P_{k+1} Y\right|\left|Y \phi_{k}(v)\right|+\left|Y v_{k+1}\right| \quad(k \geq 0) .
$$

Applying Lemma 6.1.2, we have

$$
\left|Y \phi_{k+1}(v)\right| \leq \frac{C_{3}}{(k+1)^{q}}\left|X \phi_{k}(v)\right|+\frac{C_{4}}{(k+1)^{r}}\left|Y \phi_{k}(v)\right|+\left|Y v_{k+1}\right| \quad\left(k \geq n_{0}(C)\right),
$$

and hence

$$
\begin{aligned}
& \frac{\left|Y \phi_{k+1}(v)\right|}{(k+2)^{p}} \leq \frac{C_{3}}{(k+1)^{p+q-2}} \frac{\left|X \phi_{k}(v)\right|}{(k+1)(k+2)}+\frac{C_{4}}{(k+1)^{r}} \frac{\left|Y \phi_{k}(v)\right|}{(k+1)^{p}}+\frac{\left|Y v_{k+1}\right|}{(k+2)^{p}} \\
& \leq \frac{C_{3}}{(n+1)^{p+q-2}} \frac{\left|X \phi_{k}(v)\right|}{(k+1)(k+2)}+\frac{C_{4}}{(n+1)^{r}} \frac{\left|Y \phi_{k}(v)\right|}{(k+1)^{p}}+\left|Y v_{k+1}\right| \\
& \quad\left(k \geq n \geq n_{0}(C)\right) .
\end{aligned}
$$

Summing over $k=n, n+1, n+2, \ldots$, we have

$$
g_{n+1} \leq \frac{C_{3} f_{n}}{(n+1)^{p+q-2}}+\frac{C_{4} g_{n}}{(n+1)^{r}}+\beta_{n+1} \quad\left(n \geq n_{0}(C)\right) .
$$

Applying Lemma 6.2.2, we obtain

$$
\begin{aligned}
& g_{n+1} \leq \frac{\left(1+\varepsilon C_{1}\right) C_{3}\left(C_{2} g_{n}+\alpha_{n+1}\right)}{(n+1)^{p+q-1}}+\frac{C_{4} g_{n}}{(n+1)^{r}}+\beta_{n+1} \\
&=\left\{\frac{\left(1+\varepsilon C_{1}\right) C_{2} C_{3}}{(n+1)^{p+q-1}}+\frac{C_{4}}{(n+1)^{r}}\right\} g_{n}+\frac{\left(1+\varepsilon C_{1}\right) C_{3} \alpha_{n+1}}{(n+1)^{p+q-1}}+\beta_{n+1} \\
&=\left\{\begin{array}{c}
\left(1+\varepsilon C_{1}\right) C_{2} C_{3} \\
(n+1)^{p+q-1}
\end{array} \frac{C_{4}}{(n+1)^{r}}\right\} g_{n}+\max \left\{\left(1+\varepsilon C_{1}\right) C_{3}, 1\right\}\left(\alpha_{n+1}+\beta_{n+1}\right), \\
&\left(n \geq n_{1}(C, \varepsilon)\right) .
\end{aligned}
$$

Finally, using Lemma 6.3.2, we obtain

$$
g_{n+1} \leq \frac{A g_{n}}{(n+1)^{s}}+B \gamma_{n+1} \quad(n \geq m(A, B, C)) .
$$

Corollary 6.3.4. Let $A, B$ and $m=m(A, B, C)$ be as in Lemma 6.3.3. Then,

$$
\begin{gathered}
g_{n}(v) \leq \frac{A^{n-m} g_{m}(v)}{\{(m+1)(m+2) \cdots(n-1) n\}^{s}}+\sum_{k=m+1}^{n} \frac{B A^{n-k} \gamma_{k}(v)}{\{(k+1)(k+2) \cdots(n-1) n\}^{s}} \\
\left(n \geq m, v \in \ell_{0}^{1}\right) .
\end{gathered}
$$

Proof. Induction on $n \geq m$ by using Lemma 6.3 .3 establishes this corollary.

6.4. Convexity. Given any $v \in \ell_{0}^{1}$, consider a sequence $d_{n}>0(n \geq 0)$ satisfying the following condition.

Condition 6.4.1. Let $s$ be as in Notation 3.5.4.

$$
\begin{aligned}
\frac{d_{n+1} d_{n-1}}{\left(d_{n}\right)^{2}} & \geq\left(\frac{n}{n+1}\right)^{s} & & (n \geq 1), \\
\gamma_{n}(v) & \leq d_{n} & & (n \geq 0) .
\end{aligned}
$$


Lemma 6.4.2. Let $A, B$ and $m=m(A, B, C)$ be as in Corollary 6.3.4. If $\left\{d_{n}\right\}$ satisfies Condition 6.4.1, then

$$
\begin{aligned}
g_{n}(v) & \leq \frac{A^{n-m} g_{m}(v)}{\{(m+1)(m+2) \cdots(n-1) n\}^{s}} \\
& +B(n-m) \max \left\{\frac{A^{n-m-1} d_{m+1}}{\{(m+2)(m+3) \cdots(n-1) n\}^{s}}, d_{n}\right\} \quad(n \geq m+1) .
\end{aligned}
$$

Proof. For $n \geq m+1$ we consider the sequence

$$
x_{k}=\frac{A^{n-k} d_{k}}{\{(k+1)(k+2) \cdots(n-1) n\}^{s}} \quad(m+1 \leq k \leq n) .
$$

Condition 6.4.1.(1) implies that if $n \geq m+3$, then

$$
\frac{x_{k+1} x_{k-1}}{\left(x_{k}\right)^{2}}=\left(\frac{k+1}{k}\right)^{s} \frac{d_{k+1} d_{k-1}}{\left(d_{k}\right)^{2}} \geq 1 \quad(m+2 \leq k \leq n-1) .
$$

This means that the sequence $\left\{x_{k} ; m+1 \leq k \leq n\right\}$ is multiplicatively convex (including the cases $n=m+1, m+2$ ). Hence $\left\{x_{k}\right\}$ attains the minimum at $k=m+1$ or $n$. By Corollary 6.3.4 and Condition 6.4.1(2), we have

$$
g_{n} \leq \frac{A^{n-m} g_{m}}{\{(m+1)(m+2) \cdots(n-1) n\}^{s}}+\sum_{k=m+1}^{n} x_{k} .
$$

In the sum $\sum x_{k}$, we replace each $x_{k}$ by $\max \left\{x_{k}\right\}=\max \left\{x_{m+1}, x_{n}\right\}$. Then we establish the lemma.

\subsection{Preparatory lemmas.}

Assumption 6.5.1. Assume that $(t, a)$ satisfies one of the following conditions:

(1) $t=s=0, c_{0}(|\cdot|)<a<1$,

(2) $t=0<s, 0<a<1$,

(3) $0<t<s, a>0$,

(4) $t=s>0, a>c_{0}(|\cdot|)$,

where $s$ and $c_{i}(|\cdot|)$ are defined in Notation 3.5.4 and Definition 3.5.5, respectively.

Definition 6.5.2. We set

$$
\begin{array}{ll}
L_{1}(t, a)=\frac{1}{1-a} & (t=0), \\
L_{1}(t, a)=\max \left\{2,\left(\max _{0 \leq n \leq\left[(2 a)^{1 / t}\right]} \frac{(n !)^{t}}{a^{n}}\right) \sum_{k=0}^{\infty} \frac{a^{k}}{(k !)^{t}}\right\} & (t>0) .
\end{array}
$$

Lemma 6.5.3. Under Assumption 6.5.1 we have

$$
\gamma_{n}(v) \leq L_{1}(t, a)|v|_{t, a} \frac{a^{n}}{(n !)^{t}} \quad\left(n \geq 0, v \in \mathcal{G}^{t, a}\right) .
$$

Proof. In case $t=0$ we have

$$
\gamma_{n}=\sum_{k=n}^{\infty}\left|v_{n}\right| \leq|v|_{0, a} \sum_{k=n}^{\infty} a^{k}=\frac{|v|_{0, a} a^{n}}{1-a}=L_{1}(t, a)|v|_{0, a} a^{n},
$$

which implies the lemma. In case $t>0$ we easily see that

$$
\frac{1}{2} \leq 1-\frac{a}{(k+1)^{t}} \quad\left(k \geq\left[(2 a)^{1 / t}\right]\right) .
$$


Hence we have

$$
\begin{aligned}
\left|v_{n}\right| & \leq \frac{2|v|_{t, a} a^{k}}{(k !)^{t}} \frac{1}{2} \leq \frac{2|v|_{t, a} a^{k}}{(k !)^{t}}\left\{1-\frac{a}{(k+1)^{t}}\right\} \\
& =\frac{2|v|_{t, a} a^{k}}{(k !)^{t}}-\frac{2|v|_{t, a} a^{k+1}}{\{(k+1) !\}^{t}} \quad\left(k \geq\left[(2 a)^{1 / t}\right]\right) .
\end{aligned}
$$

Summing over $k=n, n+1, n+2, \ldots$, we obtain

$$
\gamma_{n}=\sum_{k=n}^{\infty}|v| \leq \frac{2|v|_{t, a} a^{n}}{(n !)^{t}} \leq \frac{|v|_{t, a} L_{1}(t, a) a^{n}}{(n !)^{t}} \quad\left(n \geq\left[(2 a)^{1 / t}\right]\right)
$$

As for $0 \leq n \leq\left[(2 a)^{1 / t}\right]$, we have also

$$
\gamma_{n} \leq \sum_{k=0}^{\infty} \frac{|v|_{t, a} a^{k}}{(k !)^{t}}=\frac{(n !)^{t}}{a^{n}} \sum_{k=0}^{\infty} \frac{a^{k}}{(k !)^{t}} \cdot \frac{|v|_{t, a} a^{n}}{(n !)^{t}} \leq \frac{|v|_{t, a} L_{1}(t, a) a^{n}}{(n !)^{t}}
$$

This establishes the lemma.

Lemma 6.5.4. Let $\mathcal{G}_{0}^{t, a+}$ be defined by Definition 3.5.3. There exist constants $L_{2}(t, a)$ and $L_{3}(t, a)$, depending only on $(t, a)$, such that

$$
\begin{aligned}
\left|\phi_{n}(v)\right| & \leq \frac{L_{2}(t, a)}{n+1}|v|_{t, a}, \\
g_{n}(v) & \leq L_{3}(t, a)|v|_{t, a} \quad\left(n \geq 0, v \in \mathcal{G}_{0}^{t, a}\right) .
\end{aligned}
$$

Proof. By Assumption 6.5.1, the sum

$$
L_{2}^{\prime}(t, a)=\sum_{n=0}^{\infty} \frac{(n+1) a^{n}}{(n !)^{t}}+\sup _{n \geq 0} \frac{(n+1) a^{n}}{(n !)^{t}}
$$

converges and defines a finite number. By Definition 3.2.2 and Definition 3.5.1, we have $|v|_{0} \leq L_{2}^{\prime}(t, a)|v|_{t, a}$. By Theorem II (or Theorem I) and $\Phi v=0$, there exists a constant $C_{5}$ such that

$$
\left|\phi_{n}(v)\right| \leq \frac{C_{5}|v|_{0}}{n+1} \quad\left(n \geq 0, v \in \ell_{0}^{1}\right)
$$

Hence, setting $L_{2}(t, a)=C_{5} L_{2}^{\prime}(t, a)$, we obtain Assertion (1). Next, noting that $p \geq 1$, we set

$$
L_{3}(t, a)=\sum_{n=0}^{\infty} \frac{L_{2}(t, a)}{(n+1)^{p+1}}<\infty .
$$

Then we easily obtain Assertion (2). This establishes the lemma.

Remark 6.5.5. The constant $C_{5}$ in the proof depends (only) on $|\cdot| \in \mathcal{N}$. So it is better to say that the constants $L_{2}(t, a)$ and $L_{3}(t, a)$ depend only on $(t, a,|\cdot|)$. We did not refer to this dependence explicitly, since $|\cdot|$ is understood to be fixed (cf. Notation 6.1.1). 
Lemma 6.5.6. Let $A>C_{0}, B>B_{0}$ and $m=m(A, B, C)$ be as in Lemma 6.4.2. Then

$$
\begin{aligned}
& g_{n}(v) \leq L_{3}(t, a)|v|_{t, a} \frac{A^{n-m}}{\{(m+1)(m+2) \cdots(n-1) n\}^{s}} \\
&+n B L_{1}(t, a)|v|_{t, a} \max \left\{\frac{A^{n-m-1} a^{m+1}}{\{(m+1) !\}^{t}\{(m+2)(m+3) \cdots(n-1) n\}^{s}}, \frac{a^{n}}{(n !)^{t}}\right\} \\
&\left(n \geq m+1, v \in \mathcal{G}_{0}^{t, a}\right) .
\end{aligned}
$$

Proof. For any $v \in \mathcal{G}_{0}^{t, a}$ we set

$$
d_{n}=L_{1}(t, a)|v|_{t, a} \frac{a^{n}}{(n !)^{t}} \quad(n \geq 0) .
$$

Then Condition 6.4.1(1) is easily checked. Moreover, Lemma 6.5.3 implies Condition 6.4.1(2). Hence Lemma 6.4.2 is valid for the sequence $\left\{d_{n}\right\}$. The lemma easily follows from Lemma 6.4.2 and Lemma 6.5.4(2).

6.6. Gevrey estimates. We are now in a position to establish Gevrey estimates.

Lemma 6.6.1. Under Assumption 6.5.1 there exist a nonnegative integer $m(a)$ depending only on $a$ and nonnegative constants $L_{4}(t, a), L_{5}(t, a)$ depending only on $(t, a)$ such that

$$
\begin{array}{rlrl}
g_{n}(v) & \leq L_{4}(t, a)|v|_{t, a} \frac{(n+1) a^{n}}{(n !)^{t}} & & \left(n \geq m(t, a)+1, v \in \mathcal{G}_{0}^{t, a}\right), \\
f_{n}(v) & \leq L_{5}(t, a)|v|_{t, a} \frac{a^{n}}{(n !)^{t}} & \left(n \geq m(t, a)+1, v \in \mathcal{G}_{0}^{t, a}\right) .
\end{array}
$$

Proof. First we consider the cases (1) and (4) in Assumption 6.5.1. In these cases we have $t=s$ and $c_{0}(|\cdot|)<a$. By Definitions 3.5.5 and 6.3.1, we can choose the constants $C=\left(C_{1}, C_{2}, C_{3}, C_{4}\right)$ so that $C_{i}>c_{i}(|\cdot|)(i=1,2,3,4)$ and $C_{0}<a$. This choice depends only on $a$. We set $A=a\left(>C_{0}\right)$ and fix $B>B_{0}$ arbitrary. Then $(A, B, C)$ depends only on $a$. Lemma 6.5.6 implies

$$
\begin{aligned}
g_{n}(v) & \leq L_{3}(t, a)|v|_{t, a} \frac{a^{n-m}}{\{(m+1)(m+2) \cdots(n-1) n\}^{t}} \\
& +n B L_{1}(t, a)|v|_{t, a} \frac{a^{n}}{(n !)^{t}} \quad(n \geq m+1),
\end{aligned}
$$

where $m=m(A, B, C)$. If we put $m(a)=m(A, B, C)$ and $L_{4}(t, a)=B L_{1}(t, a)+$ $(m !)^{t} a^{-m} L_{3}(t, a)$, then Assertion (1) holds.

Next we consider the cases (2) and (3) in Assumption 6.5.1. In these cases we have $s<t$. We take any constants $C=\left(C_{1}, C_{2}, C_{3}, C_{4}\right)$ such that $C_{i}>c_{i}(|\cdot|)$ $(i=1,2,3,4)$. Moreover we fix $A>C_{0}$ and $B>B_{0}$ arbitrarily. Lemma 6.5.6 implies

$$
\begin{aligned}
g_{n}(v) \leq & L_{3}(t, a)|v|_{t, a} \frac{(m !)^{s}}{A^{m}} \frac{(A / a)^{n}}{(n !)^{s-t}} \cdot \frac{a^{n}}{(n !)^{t}} \\
+ & n B L_{1}(t, a)|v|_{t, a} \max \left\{\{(m+1) !\}^{s-t} \frac{(A / a)^{n-m-1}}{(n !)^{s-t}}, 1\right\} \frac{a^{n}}{(n !)^{t}} \\
& (n \geq m+1),
\end{aligned}
$$


where $m=m(A, B, C)$. So if we set $m(a)=m(A, B, C)$ and define $L_{4}(t, a)$ as below, then Assertion (1) holds, with

$$
\begin{aligned}
& L_{4}(t, a) \\
& =\sup _{n \geq m+1}\left[L_{3}(t, a) \frac{(m !)^{s}}{A^{m}} \frac{(A / a)^{n}}{(n !)^{s-t}}+B L_{1}(t, a)\left\{\{(m+1) !\}^{s-t} \frac{(A / a)^{n-m-1}}{(n !)^{s-t}}+1\right\}\right] .
\end{aligned}
$$

Since $s>t, L_{4}(t, a)$ is well-defined, i.e. $L_{4}(t, a)<\infty$.

Next, Lemmas 6.2.2, 6.5.3 and Assertion (1) imply

$$
\begin{aligned}
f_{n}(v) & \leq \frac{1+\varepsilon(A, B, C) C_{1}}{n+1}\left\{C_{2} g_{n}(v)+\gamma_{n}(v)\right\} \\
& \leq \frac{1+\varepsilon(A, B, C) C_{1}}{n+1}|v|_{t, a}\left\{C_{2} L_{4}(t, a)(n+1)+L_{1}(t, a)\right\} \frac{a^{n}}{(n !)^{t}} .
\end{aligned}
$$

Assertion (2) easily follows from this inequality.

Strictly speaking, we should say that $m(a), L_{4}(t, a)$ and $L_{5}(t, a)$ depend also on $|\cdot|$ (cf. Remark 6.5.5).

Theorem 6.6.2 (Theorem III). Under Assumptions $\mathrm{B}_{N}$ and $\mathrm{C}$, if $(t, a)$ satisfies Assumption 6.5.1 then there exists a constant $M(t, a,|\cdot|)$, depending only on $(t, a,|\cdot|)$, such that

$$
\left|\phi_{n}(v)\right| \leq M(t, a,|\cdot|)|v|_{t, a} \frac{(n+1)^{p+1} a^{n}}{(n !)^{t}} \quad\left(n \geq 0, v \in \mathcal{G}_{0}^{t, a},|\cdot| \in \mathcal{N}\right) .
$$

Proof. By Definition 6.1.3 and Lemma 6.6.1, we have

$$
\begin{aligned}
\left|\phi_{n}(v)\right| & \leq\left|Y \phi_{n}(v)\right|+\left|X \phi_{n}(v)\right| \\
\leq & (n+1)^{p} g_{n}(v)+(n+1)(n+2) f_{n}(v) \\
\leq & \left\{L_{4}(t, a)(n+1)^{p+1}+L_{5}(t, a)(n+1)(n+2)\right\}|v|_{t, a} \frac{a^{n}}{(n !)^{t}} \\
\leq & \left\{L_{4}(t, a)+2 L_{5}(t, a)\right\}|v|_{t, a} \frac{(n+1)^{p+1} a^{n}}{(n !)^{t}} \\
& \left(n \geq m(a)+1, v \in \mathcal{G}_{0}^{t, a}\right) .
\end{aligned}
$$

We set

$$
M(t, a,|\cdot|)=\max \left\{L_{4}(t, a)+2 L_{5}(t, a), L_{2}(t, a) \max _{0 \leq n \leq m(a)} \frac{(n !)^{t}}{(n+1)^{p+2} a^{n}}\right\} .
$$

Then the above inequality and Lemma 6.5.4(1) establish the theorem.

6.7. Proof of Corollary 3.6.2. To establish Corollary 3.6 .2 we show that the pair $\left(\mathcal{G}^{t, a+}, P\right)$ satisfies Conditions (AP-1)-(AP-4) in Definition 1.1. Assumption $\mathrm{B}_{\infty}$ and Definition 3.5.2 imply that $P$ and $T$ map $\mathcal{G}^{t, a+}$ into itself, and hence (AP-1). Condition (AP-2) is trivial from Definition 3.5.2. Assertion (2) of Theorem I implies (3) of (AP-3). The other conditions in (AP-3) and (AP-4) readily follow from (1) of Theorem II and Corollary 3.6.1. Hence $\left(\mathcal{G}^{t, a+}, P\right)$ is an asymptotic pair of order $N$. 


\section{EXAMPLES}

We present two simple, but nontrivial, examples to which our theory applies. These examples arise from a different area of mathematics, i.e., algebraic analysis of linear partial differential equations or the $D$-module theory. In this connection we refer to [9][10]. Let $D$ be a domain in $\mathbb{C}, \mathcal{O}(D)$ the linear space of all holomorphic functions in $D, \mathcal{K}$ the set of all compact subsets of $D$. For each $K \in \mathcal{K}$ we set

$$
|f|_{K}=\sup _{x \in K}|f(x)| \quad(f \in \mathcal{O}(D)) .
$$

Then $\mathcal{O}(D)$ is a complete locally convex linear space having semi-norms $|\cdot|_{K}$ with $K \in \mathcal{K}$.

7.1. Example 1. Let $D$ be any bounded domain in $\mathbb{C}$. We set $U=\mathcal{O}(D)^{2}$, where each element in $U$ is regarded as a column vector. Let $\beta$ and $\gamma$ be complex constants such that $\gamma \notin \mathbb{Z}$. We consider the difference equation (1.1), where

$$
P_{n}=\left(\begin{array}{cc}
1 & \frac{-x}{n-\gamma} \\
\frac{-\beta}{n-\gamma} & \frac{-x}{n-\gamma}
\end{array}\right) .
$$

This example arises from the confluent hypergeometric system of two variables known as the Humbert system $\Phi_{2}$ :

$$
\left\{\begin{array}{l}
{\left[x \partial_{x}^{2}+y \partial_{x} \partial_{y}+(\gamma-x) \partial_{x}-\beta\right] u=0} \\
{\left[y \partial_{y}^{2}+x \partial_{x} \partial_{y}+(\gamma-y) \partial_{y}-\beta^{\prime}\right] u=0}
\end{array}\right.
$$

where $\beta, \beta^{\prime}, \gamma \in \mathbb{C}$. We refer to [9] for the derivation of (7.2) from $\left(\Phi_{2}\right)$. From (7.2) we have $P_{n}=P^{(0)}+P^{(1)}(n-\gamma)_{1}$, where

$$
P^{(0)}=\left(\begin{array}{ll}
1 & 0 \\
0 & 0
\end{array}\right), \quad P^{(1)}=\left(\begin{array}{ll}
0 & x \\
\beta & x
\end{array}\right), \quad P^{(i)}=O \quad(i \geq 2) .
$$

As the supplementary projections $X$ and $Y$, we take

$$
X=\left(\begin{array}{ll}
1 & 0 \\
0 & 0
\end{array}\right), \quad Y=\left(\begin{array}{ll}
0 & 0 \\
0 & 1
\end{array}\right) .
$$

Then we have (cf. Notation 3.2.1)

$$
U_{\nu}=\mathcal{O}(D) e_{\nu} \quad(\nu=0,1),
$$

where $e_{\nu} \in U$ are given by

$$
e_{0}=\left(\begin{array}{l}
0 \\
1
\end{array}\right), \quad e_{1}=\left(\begin{array}{l}
1 \\
0
\end{array}\right) .
$$

Any $f \in U$ is expressed as $f=f_{0} e_{0}+f_{1} e_{1}$ for some $f_{0}, f_{1} \in \mathcal{O}(D)$. For each $K \in \mathcal{K}$ let $|f|_{K}=\sqrt{\left|f_{0}\right|_{K}^{2}+\left|f_{1}\right|_{K}^{2}}$ (cf. (7.1)). We take $\mathcal{N}=\left\{|\cdot|_{K} ; K \in \mathcal{K}\right\}$ as the system of semi-norms on $U$. Then the condition (2.1) holds. By using the assumption that $D$ is bounded, it is easily checked that Assumption $\mathrm{A}_{u}$ is satisfied for any $\rho$ such that $0<\rho<1$. Moreover, Assumption $\mathrm{B}_{\infty}$ is also satisfied.

Notation 3.2.4, (7.3) and (7.4) yield

$$
P_{i j}= \begin{cases}\frac{P^{(1)}}{j_{+}} & (i=j+1), \\ O & (i>j+1),\end{cases}
$$


where $j_{+}=\max \{j, 1\}$. Notation 3.2.5, (7.3), (7.4) and (7.6) yield

$$
A_{i j}=\left\{\begin{array}{cl}
\frac{1}{j_{+}}\left(\begin{array}{cc}
\frac{\beta x}{i} & \frac{x^{2}}{i}, \\
\beta & x
\end{array}\right) & (i=j+1), \\
O & (i>j+1) .
\end{array}\right.
$$

Since $A_{i j}=O$ for $i>j+1$, we have $A_{J}=O$ for any $J \in \mathbf{S}_{i}$ other than $J=$ $\{1,2, \ldots, i-1\}$ (cf. Definition 3.2.6). Hence $\Psi_{i}=A_{i, i-1} A_{i-1, i-2} \cdots A_{21} A_{10}$ for $i \geq 1$. Using (7.7) we can show that the operators $\Psi_{i}$ in Definition 3.2.6 are expressed as

$$
\Psi_{i}=\frac{(\beta+1)(\beta+2) \cdots(\beta+i-1)}{[(i-1) !]^{2}}\left(\begin{array}{cc}
\frac{\beta x^{i}}{i} & \frac{x^{i+1}}{i} \\
\beta x^{i-1} & x^{i}
\end{array}\right) \quad(i \geq 1) .
$$

We set $p=q=r=1$. Then Assumption $\mathrm{C}$ is satisfied and we have $s=1$ in Notation 3.5.4. Using Remark 3.5.6 we have (cf. Definition 3.5.5)

$$
c_{2}\left(|\cdot|_{K}\right)=c_{4}\left(|\cdot|_{K}\right)=r_{K}, \quad c_{3}\left(|\cdot|_{K}\right)=|\beta| \quad(K \in \mathcal{K}),
$$

where $r_{K}=\sup _{x \in K}|x|$. Hence $c_{0}\left(|\cdot|_{K}\right)=r_{K}(|\beta|+1)$. Therefore the constant $a_{0}$ in Definition 3.5.5 is given by

$$
a_{0}=r_{D}(|\beta|+1), \quad \text { where } \quad r_{D}=\sup _{x \in D}|x| .
$$

From Corollaries 3.4.2 and 3.6.2 we obtain the following proposition.

Proposition 7.1. Let $P=\left(P_{n}\right)$ be as in (7.2) and $U=\mathcal{O}(D)^{2}$, where $D$ is a bounded domain in $\mathbb{C}$. Assume that $V=\ell^{\infty}$ or $\mathcal{G}^{t, a+}$, where $(t, a)$ satisfies one of the following conditions:

(1) $t=0,0 \leq a<1$

(2) $0<t<1, a \geq 0$,

(3) $t=1, a \geq a_{0}$, where $a_{0}$ is given by (7.9).

Then $(V, P)$ is an asymptotic pair of order infinity with asymptotic data $\mathcal{A}=$ $\left(-\gamma, U_{1},\left\{\Psi_{i}\right\}_{i=0}^{\infty}\right)$, where $U_{1}$ and $\Psi_{i}$ are given by (7.5) and (7.8), respectively.

7.2. Example 2. Let $\mathbb{D}=\{x \in \mathbb{C} ;|x|<1\}$ be the open unit disk in $\mathbb{C}, D$ any relatively compact domain in $\mathbb{D}$. We set $U=\mathcal{O}(D)^{2}$, where each element in $U$ is regarded as a column vector. Let $\alpha, \beta$ and $\gamma$ be complex constants such that $\gamma \notin \mathbb{Z}$. We consider the difference equation (1.1), where

$$
P_{n}=\left(\begin{array}{cc}
1+\frac{\beta x}{n-\gamma} & \frac{x(x-1)}{n-\gamma} \\
\frac{\beta(n-\alpha)}{n-\gamma} & \frac{x(n-\alpha)}{n-\gamma}
\end{array}\right)
$$

This example arises from the confluent hypergeometric system of two variables known as the Humbert system $\Phi_{1}$ :

$$
\left\{\begin{array}{l}
{\left[x(1-x) \partial_{x}^{2}+y(1-x) \partial_{x} \partial_{y}+\{\gamma-(\alpha+\beta+1) x\} \partial_{x}-\beta y \partial_{y}-\alpha \beta\right] u=0,} \\
{\left[y \partial_{y}^{2}+x \partial_{x} \partial_{y}+(\gamma-y) \partial_{y}-x \partial_{x}-\alpha\right] u=0}
\end{array}\right.
$$


We refer to [9] for the derivation of (7.10) from $\left(\Phi_{1}\right)$. From (7.10) we have $P_{n}=$ $P^{(0)}+P^{(1)}(n-\gamma)_{1}$, where

$$
P^{(0)}=\left(\begin{array}{cc}
1 & 0 \\
\beta & x
\end{array}\right), \quad P^{(1)}=\left(\begin{array}{cc}
-\beta x & x(1-x) \\
\beta(\alpha-\gamma) & (\alpha-\gamma) x
\end{array}\right), \quad P^{(i)}=O \quad(i \geq 2) .
$$

As the supplementary projections $X$ and $Y$, we take

$$
X=\left(\begin{array}{cc}
1 & 0 \\
\frac{\beta}{1-x} & 0
\end{array}\right), \quad Y=\left(\begin{array}{cc}
0 & 0 \\
\frac{-\beta}{1-x} & 1
\end{array}\right) .
$$

Then we have (cf. Notation 3.2.1)

$$
U_{\nu}=\mathcal{O}(D) e_{\nu} \quad(\nu=0,1)
$$

where $e_{\nu} \in U$ are given by

$$
e_{0}=\left(\begin{array}{l}
0 \\
1
\end{array}\right), \quad e_{1}=\left(\begin{array}{c}
1 \\
\frac{\beta}{1-x}
\end{array}\right) .
$$

Any $f \in U$ is expressed as $f=f_{0} e_{0}+f_{1} e_{1}$ for some $f_{0}, f_{1} \in \mathcal{O}(D)$. For each $K \in \mathcal{K}$ let $|f|_{K}=\sqrt{\left|f_{0}\right|_{K}^{2}+\left|f_{1}\right|_{K}^{2}}$ (cf. (7.1)). We take $\mathcal{N}=\left\{|\cdot|_{K} ; K \in \mathcal{K}\right\}$ as the system of semi-norms on $U$. Then the condition (2.1) holds. We set

$$
\rho=r_{D}:=\sup _{x \in D}|x| \text {. }
$$

Since $D$ is relatively compact in $\mathbb{D}$, we have $0 \leq \rho<1$. Assumption $\mathrm{A}_{u}$ is satisfied for $\rho$ defined by (7.14). Moreover Assumption $\mathrm{B}_{\infty}$ is also satisfied. We only check (2) of Assumption $\mathrm{B}_{\infty}$. The operator $Z$ is expressed as

$$
Z:=Y P^{(0)} Y=\left(\begin{array}{cc}
0 & 0 \\
\frac{-\beta x}{1-x} & x
\end{array}\right) \text {. }
$$

This implies $Z e_{0}=x e_{0}$ and $Z e_{1}=0$, i.e., $Z$ is the multiplication by $x$ on $U_{0}$ and zero on $U_{1}$. In particular we have

$$
|Z|_{K}=r_{K}:=\sup _{x \in K}|x|<1 \quad(\forall K \in \mathcal{K}) .
$$

This implies (2) of Assumption $\mathrm{B}_{\infty}$.

Notation $3.2 .4,(7.11)$ and $(7.12)$ yield

$$
P_{i j}= \begin{cases}\frac{P^{(1)}}{j_{+}} & (i=j+1), \\ O & (i>j+1) .\end{cases}
$$

Notation 3.2.5, (7.11), (7.12), (7.15) and (7.17) yield

$$
A_{i j}= \begin{cases}\frac{1}{j_{+}}\left(\frac{x}{1-x}\right) Q W Q^{-1} & (i=j+1), \\ O & (i>j+1),\end{cases}
$$

where the matrices $Q$ and $W$ are given by

$$
Q=\left(\begin{array}{cc}
1 & 0 \\
\frac{\beta}{1-x} & 1
\end{array}\right), \quad W=\left(\begin{array}{cc}
\frac{\beta(\alpha-\gamma)}{i} & \frac{\left(\alpha-\beta-\gamma-j_{+}\right) x(1-x)}{i} \\
\frac{\beta(\alpha-\gamma)}{x(1-x)} & \alpha-\beta-\gamma-j_{+}
\end{array}\right)
$$


Since $A_{i j}=O$ for $i>j+1$, we have $A_{J}=O$ for any $J \in \mathbf{S}_{i}$ other than $J=$ $\{1,2, \ldots, i-1\}$ (cf. Definition 3.2.6). Hence

$$
\Psi_{i}=A_{i, i-1} A_{i-1, i-2} \cdots A_{21} A_{10}
$$

for $i \geq 1$. Using (7.18) we can show that the operators $\Psi_{i}$ in Definition 3.2.6 are expressed as

$$
\begin{aligned}
\Psi_{i} & =\left(\frac{x}{1-x}\right)^{i} \frac{(\beta+1)(\beta+2) \cdots(\beta+i-1) \cdot(\alpha-\gamma-1)(\alpha-\gamma-2) \cdots(\alpha-\gamma-i+1)}{[(i-1) !]^{2}} \\
& \times\left(\begin{array}{cc}
\frac{\beta\{\alpha-\gamma-(\alpha-\beta-\gamma-1) x\}}{i} & \frac{(\alpha-\beta-\gamma-1) x(1-x)}{i} \\
\frac{\beta\{\alpha-\gamma-(\alpha-\beta-\gamma-1) x\}}{x(1-x)}\left(\frac{\beta x}{i}+1\right) & (\alpha-\beta-\gamma-1)\left(\frac{\beta x}{i}+1\right)
\end{array}\right) .
\end{aligned}
$$

We set $p=q=1$ and $r=0$. Then Assumption $\mathrm{C}$ is satisfied and we have $s=0$ in Notation 3.5.4. By Remark 3.5.6 and (7.16), we have $c_{0}\left(|\cdot|_{K}\right)=|Z|_{K}=r_{K}$ for any $K \in \mathcal{K}$. Hence (7.14) implies that the constant $a_{0}$ in Definition 3.5.5 is given by

$$
a_{0}=r_{D}<1 .
$$

From Corollaries 3.4.2, 3.6.2 and (7.20) we obtain the following proposition.

Proposition 7.2. Let $P=\left(P_{n}\right)$ be as in (7.10) and $U=\mathcal{O}(D)^{2}$, where $D$ is a relatively compact domain in the open unit disk $\mathbb{D}$. Let a be any constant such that $r_{D} \leq a<1$, where $r_{D}$ is defined by (7.14). Assume that $V=\ell^{\infty}$ or $\mathcal{G}^{0, a+}$. Then $(V, P)$ is an asymptotic pair of order infinity with asymptotic data $\mathcal{A}=\left(-\gamma, U_{1},\left\{\Psi_{i}\right\}_{i=0}^{\infty}\right)$, where $U_{1}$ and $\Psi_{i}$ are given by (7.13) and (7.19), respectively.

\section{REFERENCES}

1. Benzaid, Z. and Lutz, D.A., Asymptotic representation of solutions of perturbed systems of linear difference equations, Studies in Appl. Math. 77 (1987), 195-221. MR 90f:39003

2. Birkhoff, G.D., General theory of linear difference equations, Trans. Amer. Math. Soc. 12 (1911), 243-284.

3. Charrière, H. and Gérard, R., The rings of formal and convergent inverse factorial series, Kumamoto J. Math. 5 (1992), 1-20. MR 94b:39019

4. Coffman, C.V., Asymptotic behavior of solutions of ordinary difference equations, Trans. Amer. Math. Soc. 110 (1964), 22-51. MR 27:6054

5. Evgrafov, M.A., On the asymptotic behavior of solutions of difference equations (in Russian), Dokl. Akad. Nauk SSSR 121 (1958), 26-219. MR 21:5102

6. Gérard, R. and Lutz, D.A., Convergent factorial series solutions of singular operator equations, Analysis 10 (1990), 99-145. MR 92f:39005

7. Hirai, I. and Yanagihara, N., Difference equations in Banach spaces, Comm. Math. Univ. St. Paul 28 (1980), 51-61. MR 81k:39002

8. Ishizuka, S., On solution complexes of D-modules associated with confluent hypergeometric differential equations, (in Japanese), Master thesis, Ochanomizu University, Tokyo, 1994.

9. Ishizuka, S., Iwasaki, K. and Majima, H., Gevrey cohomology groups for the Humbert systems, in preparation.

10. Iwasaki, K., Cohomology groups for recurrence relations, Preprint (1996).

11. Li, Z.-H., The asymptotic estimates of solutions of difference equations, J. Math. Anal. Appl. 94 (1983), 181-192. MR 94g:39001 
12. Milne-Thomson, L.M., The calculus of finite differences, Chelsea Publ., New York, 1981. MR 13:245c (2nd ed.)

13. Nielsen, N., Recherches sur les séries de factorielles, Ann. Ecole Norm. Sup. 19 (1902), 409453.

Department of Mathematical Sciences, The University of Tokyo, 3-8-1 Komaba, Meguro-Ku, TOKYO 153 JAPAN

Current address: Department of Mathematics, Kyushu University, G-10-1 Hakozaki, Higashiku, Fukuoka 812-81 Japan

E-mail address: iwasaki@ms.u-tokyo-ac.jp 